\title{
Structural basis of oligomerization in the stalk region of dynamin-like MxA
}

Song Gao ${ }^{1,2}$, Alexander von der Malsburg ${ }^{3}$, Susann Paeschke ${ }^{1}$, Joachim Behlke ${ }^{1}$, Otto

Haller $^{3}$, Georg Kochs ${ }^{3}$, Oliver Daumke ${ }^{1,4}$

${ }^{1}$ Max-Delbrück-Centrum for Molecular Medicine, Crystallography, Robert-Rössle-

Strasse 10, 13125 Berlin, Germany

${ }^{2}$ Institute for Chemistry and Biochemistry, Free University Berlin, Takustrasse 3, 14195

Berlin, Germany

${ }^{3}$ Department of Virology, Institute for Medical Microbiology and Hygiene, University of Freiburg, Hermann-Herder-Strasse 11, 79104 Freiburg, Germany

${ }^{4}$ Institute of Medical Physics and Biophysics, Charité, Ziegelstrasse 5-9, 10117 Berlin, Germany 
The interferon-inducible dynamin-like MxA (Myxovirus resistance protein 1) GTPase is a key mediator of cell-autonomous innate immunity against livethreatening pathogens such as influenza viruses ${ }^{1}$. MxA partially localises to COPI-positive membranes of the smooth endoplasmic reticulum-Golgi intermediate compartment ${ }^{2}$. Upon infection, it redistributes to sites of viral replication and promotes missorting of essential viral constituents ${ }^{3,4}$. It has been proposed that the middle domain (MD) and the GTPase effector domain (GED) of dynamin-like GTPases constitute a stalk which mediates oligomerization and transmits conformational changes from the G-domain to the target structure ${ }^{5-7}$, but the molecular architecture of this stalk remained elusive. Here, we report the crystal structure of the stalk of MxA which folds into a four-helical bundle. This structure tightly oligomerises in the crystal in a criss-cross pattern involving three distinct interfaces and one loop. Mutations in each of these interaction sites interfere with native assembly, oligomerization, membrane binding and antiviral activity of MxA. Based on these results, we propose a structural model for dynamin oligomerization and stimulated GTP hydrolysis that is consistent with previous structural predictions and has functional implications for all members of the dynamin family.

His-tagged full-length human MxA (Fig. 1a) was recombinantly expressed in bacteria and purified to homogeneity (Methods, Supp. Fig. 1). In crystallization trials, small needle-shaped protein crystals were obtained which represented proteolytic cleavage products of the MD and GED (Supp. Fig. 2). We solved the phase problem by a single anomalous dispersion protocol and could build and refine a model containing two molecules in the asymmetric unit (Methods, Supp. Table 1 and 2). 
Each monomer spans nearly the complete MD and the amino(N-)-terminal part of the GED (amino acids 366-633) which together fold into an elongated anti-parallel fourhelical bundle where the MD contributes three helices and the GED one (Fig. 1b, Supp. Fig. 3). This segment corresponds to the stalk region of dynamin ${ }^{7}$, and we refer to it as stalk of MxA. The first visible amino acid, Glu366, is 15 amino acids downstream of the last visible residue of the corresponding G-domain structure in rat dynamin (Supp. Fig. 3) ${ }^{8}$. It marks the start of helix $\alpha 1$ in the MxA stalk which is divided in $\alpha 1^{\mathrm{N}}$ and $\alpha 1^{\mathrm{C}}$ by a 10 amino acid long loop, L1, introducing a $30^{\circ} \mathrm{kink}$. A putative loop L2 (amino acids 438-447) opposite of the deduced position of the G-domain is not visible in our structure. L2 was previously demonstrated to be the target of a functionally neutralising monoclonal antibody ${ }^{9,10}$. Helix $\alpha 2$ runs anti-parallel to $\alpha 1$ back to the G-domain. It ends in a short loop L3 and is followed by helix $\alpha 3$ that extends in parallel to $\alpha 1$. The 40 amino acid long loop L4 (residues 532-572) is at the equivalent sequence position as the PH domain of dynamin (Fig. 1a, Supp. Fig. 3) and is absent in our model. L4 is predicted to be unstructured and was previously shown to be proteinase $\mathrm{K}$ sensitive ${ }^{11}$. At the C-terminus, the GED supplies 44 residues to helix $\alpha 4$ which proceeds in parallel to helix $\alpha 2$ back to the G-domain. It is followed by a short helix $\alpha 5$ which directs the polypeptide chain towards the $\mathrm{N}$-terminus of the $\mathrm{MD}$. The carboxy(C-)-terminal 30 highly conserved residues of the GED known to be involved in antiviral specificity ${ }^{12}$ are missing in our model. In dynamin, the corresponding residues were shown to directly interact with the G-domain ${ }^{13}$. The stalk of MxA is divergent from the corresponding structures of other dynamin superfamily members, such as GBP $1^{14}, \mathrm{EHD} 2^{15}$ and $\mathrm{BDLP}^{16}$ although some features are shared (Supp. Fig. 4). 
In the crystal lattice, each MxA stalk is assembled in a criss-cross pattern resulting in a linear oligomer, where each stalk contributes three distinct interfaces (Fig. 1c). Such an arrangement of the stalks is plausible for the Mx oligomer since all G-domains would be located at one side of the oligomer whereas the putative substrate-binding site in L2 and L4 would be located at the opposite side (Fig. 1b, c).

The hydrophobic interface-1 covering $1300 \AA^{2}$ is conserved among Mx proteins and dynamins and has a two-fold symmetry between the associating monomers (Fig. 1d, Supp. Fig. 3, Supp. Fig. 5). Analytical ultracentrifugation (AUC) experiments showed that wild-type (wt) MxA was a stable tetramer (Fig. 2a, Supp. Fig. 6a), similar to dynamin $^{17,18}$. Strikingly, mutations in interface-1 (L617D, D377K, K614D, L620D, I376D) led to the disruption of the tetramer, resulting predominantly in dimers (Fig. 2a, Supp. Fig. 6b). These mutants eluted slightly later in analytical gel filtration experiments than the wt protein (Supp. Fig. 6c).

The hydrophobic interface- 2 is $1700 \AA^{2}$ in size and also has a two-fold symmetry (Fig. 1e). It is nearly invariant in Mx proteins but shows only limited sequence similarity to dynamins (Supp. Fig. 3, Supp. Fig. 5). Individual mutations M527D and F602D in interface-2 led to a complete disruption of the tetramer, resulting in a predominantly monomeric form (Fig. 2a, Supp. Fig. 6a, b, c).

Interface-3 covering 400-500 $\AA^{2}$ is non-symmetric and mediates lateral contacts between stalks oriented in parallel (Fig. 1f). Residues in this interface show higher temperature factors compared to residues in interface-1 and 2 (Supp. Fig. 5d), suggesting increased flexibility of this interface in the linear oligomer. Interface-3 involves loop L1 which interacts with residues in helix $\alpha 2$ of a neighbouring stalk. 
Furthermore, the surface-exposed Arg408 in helix $\alpha 1^{C}$, completely conserved in $\mathrm{Mx}$ proteins, is in vicinity of loop L2 of a neighbouring monomer which features an invariant ${ }^{440}$ YRGRE motif. Mutation R408D in $\alpha 1^{\mathrm{C}}$ and a quadruple mutation in this motif to alanine promoted disruption of the tetramer and the formation of a stable dimer (Fig. 2a, Supp. Fig. 6a, b, c). Remarkably, the R361S and R399A mutations in dynamin are located at equivalent positions (Supp. Fig. 3), respectively, and induce formation of stable dimers as well ${ }^{18}$. Also mutation G392D in L1 of MxA led to the disruption of the tetramer into a stable dimer (Supp. Fig. 6b). Interestingly, the corresponding mutation in yeast dynamin-like DNM1, G385D, has a similar phenotype ${ }^{19}$.

Loop L4 is in vicinity of the corresponding loop L4 from an opposing molecule (Fig. 1f) and might represent another low affinity interaction site. Confirming this hypothesis, deletion of residues 533-561 within this loop ( $\Delta \mathrm{L} 4$ mutant) resulted in a stable dimer (Fig. 2a, Supp. Fig. 6c).

MxA reversibly forms ring and spiral-like oligomers at low salt concentration or protein concentrations $>1.5 \mathrm{mg} / \mathrm{ml}$ which can be sedimented by high-speed ultracentrifugation $^{20}$. Accordingly, approximately $50 \%$ of wt MxA was sedimented in the absence of nucleotides at a protein concentration of $2.3 \mathrm{mg} / \mathrm{ml}$ (Fig. 2b). Nearly all wt MxA was found in the pellet fraction when GTP- $\gamma-\mathrm{S}$ was added at a saturating concentration. In contrast, mutants in interface-1 (L617D, K614D, L620D, I376D), interface-2 (M527D, F602D) and the $\Delta \mathrm{L} 4$ mutant could not be sedimented by ultracentrifugation, irrespective of the presence or absence of nucleotide, indicating that these interaction sites are critical for oligomerization (Fig. 2b, Supp. Fig. 6d). Mutants in interface-3 (R408D, YRGR440-443AAAA, G392D) and the D377K mutant in 
interface-1 oligomerised with reduced efficiency only in the presence of GTP- $\gamma$-S (Fig. 2b, Supp. Fig. 6d).

To examine the role of the four interaction sites for self-assembly in vivo, a nuclear accumulation assay was used which employs an artificial nuclear form of MxA carrying a foreign nuclear localization signal (NLS) and an HA-tag for detection (HA-TMxA) ${ }^{21}$. When expressed alone, FLAG-tagged wt MxA showed mostly cytoplasmic localization (Fig. 2c, Supp. Fig. 7). However, upon co-expression with the nuclear form of MxA, it accumulated predominantly in the nucleus. HA-TMxA constructs with mutations in either interface-1 (L617D), interface-3 (R408D) or loop L4 ( $\Delta$ L4) were still able to promote nuclear accumulation of wt MxA in the nucleus. In contrast, the interface-2 mutant M527D had lost this capacity. We conclude that mutants in interface-1, 3 and L4 retain the ability to form dimers with wt MxA in vivo, whereas mutations in interface-2 are disruptive. This was also confirmed in co-immunoprecipitation assays (Supp. Fig. 8).

MxA binds to liposomes and induces liposome tubulation, similarly to dynamin ${ }^{22,23}$. Interestingly, none of the MxA mutants showed significant binding to liposomes (Supp. Fig. 9), indicating that liposome binding requires a self-assembly competent MxA molecule in which all three interfaces and L4 are intact.

GTPase assays with representative mutants were performed using multiple-turnover assays (excess of GTP over MxA). When incubated with saturating concentrations of $\mathrm{GTP}^{24,25}$, wt MxA and the R408D mutant (which can still partly oligomerise in the presence of GTP- $\gamma-\mathrm{S}$, see Fig. 2b) showed a protein concentration-dependent specific GTPase rate with an estimated $k_{\max }$ of $6 \mathrm{~min}^{-1}$ (Fig. 2d), indicating that GTP hydrolysis 
is stimulated by a cooperative mechanism. The GTPase activity of wt MxA did not change significantly in the presence of liposomes. Surprisingly, the dimeric mutants L617D and $\Delta \mathrm{L} 4$ had a 3- to 4-fold increased GTPase rate at higher protein concentration ( $3 \mathrm{mg} / \mathrm{ml}$ ) and the monomeric M527D mutant an even 6-fold increased rate, whereas the GTPase rates at protein concentrations below $0.5 \mathrm{mg} / \mathrm{ml}$ were comparable to wt MxA (Fig. 2d). Nucleotide binding studies revealed that wt MxA and M527D bound with similar affinities to GDP $\left(\mathrm{K}_{\mathrm{d}}=16\right.$ and $18 \mu \mathrm{M}$, respectively), whereas wt MxA had a 3-fold higher apparent affinity to a non-hydrolysable GTP analogue, GMPPNP $\left(\mathrm{K}_{\mathrm{d}}=2.3\right.$ and $6.8 \mu \mathrm{M}$, respectively, Supp. Fig. 10a, b). The higher apparent affinity was caused by a slower off-rate of GMPPNP (Supp. Fig. 10c, d), indicating that nucleotide release might be restrained by tetramerization / higher-order oligomerization via the stalk (Supp. Fig. 10). We conclude that oligomerization of MxA via the stalk region is not a prerequisite for assembly-stimulated GTP hydrolysis but influences nucleotide-release in the G-domain.

LaCrosse virus (LACV) is an important cause of pediatric encephalitis in North America. MxA blocks its replication by sequestering the viral nucleocapsid protein into perinuclear deposits ${ }^{4,26}$. In contrast to wt MxA, the assembly-defective mutants showed no co-localization and the viral proteins accumulated near the Golgi compartment where infectious viral particles are formed (Fig. 3a, Supp. Fig. 11). We conclude that each of the four interaction sites is required for recognition of viral structures.

Next, inhibition of the polymerase complex of a highly pathogenic H5N1 influenza virus (isolated from a fatal human case in Vietnam ${ }^{27}$ ) was assessed in a mini-replicon reporter assay ${ }^{28}$. As previously shown, wt MxA inhibited viral replication by $80 \%$ (Fig. 
3b). Mutations in each of the three interfaces and L4 completely abrogated antiviral activity. These results from two independent assays indicate that proper assembly of the MxA stalk region is essential for the antiviral function.

The MxA stalks are assembled in linear oligomers in our crystals whereas previous EM studies of full-length MxA and dynamin revealed the formation of ring-like oligomers of various diameters inducing liposome tubulation ${ }^{20,22,23}$. We reasoned that the basic building block of an MxA oligomer contains a stable stalk dimer assembled via interface-2. We combined these MxA stalk dimers with structural models of the Gdomain and $\mathrm{PH}$ domain of dynamin and fitted them into the electron density map of oligomerised dynamin, obtained by cryo-EM reconstruction ${ }^{5}$ (Fig. 4a, b, Supp. Fig. 12a, b). To accomplish formation of a helical turn, a $28^{\circ}$ rotation around the centre of interface-1 was introduced between adjacent stalk dimers (Fig. 4a). All residues in interface-1 shown to be crucial for oligomerization (Fig. 2b, Supp. Fig. 6d) were maintained in the interface by this rotation (Supp. Fig. 12c, d). However, the proposed rotation moves interface-3 residues from neighbouring monomers closer towards each other (Fig. 4a). The identified oligomerization sites in interface-3 include two loop regions (L1, L2) which, together with the proposed interaction in L4, might allow some flexibility in the degree of rotation, concomitant with varying ring diameters as observed for oligomerised dynamin and $\mathrm{MxA}^{23,29}$ (Fig. 4c). Our oligomeric model features a criss-cross arrangement of the stalks (Fig. 4a, c) and accounts for the T-bar shape seen in side-views of oligomerised dynamin ${ }^{5}$ and $\operatorname{MxA}^{23}$ (Fig. 4b). Furthermore, it explains the connectivity of the G-domain with the $\mathrm{PH}$ domain in oligomerised dynamin and is in agreement with the formation of a "bundle signalling element" between the G-domain and the C-terminal part of the GED ${ }^{13}$ (Fig. 4a, Supp. Fig. 12e). 
The highly conserved surface patches in the G-domains across the nucleotide binding sites are pointing away from the central stalk of the ring and are not in contact with other G-domains of the same ring (Fig. 4b, c). Consequently, our model suggests that the G-domains are not involved in ring formation but facilitate inter-ring contacts (see also ref. 5), resulting in assembly-stimulated nucleotide hydrolysis, as demonstrated for $\mathrm{GBP}^{30}$ and proposed for $\mathrm{EHD} 2^{15}$ and $\mathrm{BDLP}{ }^{16}$. In case of MxA and dynamin, such architecture implies that GTP hydrolysis is only stimulated after formation of one complete helical turn so that G-domains from neighbouring turns can approach each other (Fig. 4c).

By presenting a molecular model for oligomerization in the stalk region of the dynamin family we provide the structural framework to understand the mechanism of membrane fission in dynamin and of the antiviral activity in MxA.

\section{Methods Summary}

Oligomerization assays were carried out at $2.3 \mathrm{mg} / \mathrm{ml}$ protein concentration in the absence and presence of $1 \mathrm{mM} \mathrm{GTP-} \gamma-\mathrm{S}$. Samples were incubated at room temperature for $10 \mathrm{~min}$ in a buffer containing $20 \mathrm{mM}$ HEPES (pH 7.5), $300 \mathrm{mM} \mathrm{NaCl}$ and $2 \mathrm{mM}$ $\mathrm{MgCl}_{2}$. After ultracentrifugation at $200,000 \mathrm{~g}, 25^{\circ} \mathrm{C}$ for $10 \mathrm{~min}$, equivalent amounts of supernatant and pellet were loaded on SDS-PAGE. Liposome co-sedimentation assays were carried out as described (www.endocytosis.org). Initially, the salt concentration was optimised that wt MxA was not sedimented in the absence of liposomes. The final reaction conditions were $0.75 \mathrm{mg} / \mathrm{ml} \mathrm{MxA}$ protein and $0.5 \mathrm{mg} / \mathrm{ml}$ unfiltered Folch liposomes fraction I (Sigma) in $20 \mathrm{mM}$ HEPES (pH 7.5), $300 \mathrm{mM} \mathrm{NaCl}$, and a 100,000 
$\mathrm{g}$ spin for $20 \mathrm{~min}$ at $25^{\circ} \mathrm{C}$. Results shown are representative for three independent experiments.

Supplementary Information is linked to the online-version of the paper at www.nature.com/nature.

Acknowledgements This project was supported by a grant of the Deutsche Forschungsgemeinschaft (SFB 740-From Molecules to Modules) and by a Career Development Fellowship of "The International Human Frontier Science Program Organization" to O.D., and by the German-Israeli-Research foundation (GIF-841/04) to G.K. and O.H. We are grateful to J. Hinshaw and J. Mears for providing us the EM maps and model fittings of oligomerised dynamin. We also would like to acknowledge help and support of O. Ristau and K. Schilling/Nanolytics (analytical ultracentrifugation), M. Dahte and H. Nikolenko (fluorescence measurements), A. Herrmann, T. Korte and P. Müller (stopped-flow analysis), G. Dittmar (mass spectrometry analysis) and the BESSY staff at BL14.1 (data collection). This work was conducted by A.v.M. in partial fulfilment for a PhD degree from the Faculty of Biology at the University of Freiburg, Germany.

Authors' contribution S.G. solved the structure and carried out the biochemical characterization of MxA mutants. A.v.M. carried out all antiviral and cellular assays. S.P. assisted S.G. in cloning and purification. J.B. performed the analytical ultracentrifugation analysis. S.G., A.v. M., O.H., G.K. and O.D. planned the experimental design and wrote the manuscript.

Authors' information The atomic coordinates of the MxA stalk have been deposited in the Protein Data Bank with accession number 3LJB. Reprints and permission information is available at npg.nature.com/reprintsandpermissions. The authors declare no competing financial interest. Correspondence and requests for materials should be addressed to O.D. (oliver.daumke@mdc-berlin.de). 


\section{References}

1 Haller, O., Stertz, S., and Kochs, G. The Mx GTPase family of interferon-induced antiviral proteins. Microbes. Infect. 9, 1636-1643 (2007)

2 Stertz, S., et al. Interferon-induced, antiviral human MxA protein localizes to a distinct subcompartment of the smooth endoplasmic reticulum. J. Interferon Cytokine Res. 26, 650-660 (2006)

3 Kochs, G. and Haller, O. Interferon-induced human MxA GTPase blocks nuclear import of Thogoto virus nucleocapsids. Proc. Natl Acad. Sci. U. S. A 96, 2082-2086 (1999)

4 Reichelt, M., et al. Missorting of LaCrosse virus nucleocapsid protein by the interferoninduced MxA GTPase involves smooth ER membranes. Traffic. 5, 772-784 (2004)

5 Mears, J. A., Ray, P., and Hinshaw, J. E. A corkscrew model for dynamin constriction. Structure. 15, 1190-1202 (2007)

6 Klockow, B., et al. The dynamin A ring complex: molecular organization and nucleotidedependent conformational changes. EMBO J. 21, 240-250 (2002)

7 Chen, Y. J., et al. The stalk region of dynamin drives the constriction of dynamin tubes. Nat. Struct. Mol. Biol. 11, 574-575 (2004)

8 Reubold, T. F., et al. Crystal structure of the GTPase domain of rat dynamin 1. Proc. Natl Acad. Sci. U. S. A 102, 13093-13098 (2005)

9 Flohr, F., et al. The central interactive region of human MxA GTPase is involved in GTPase activation and interaction with viral target structures. FEBS Lett. 463, 2428 (1999)

10 Arnheiter, H. and Haller, O. Antiviral state against influenza virus neutralized by microinjection of antibodies to interferon-induced Mx proteins. EMBO J 7, 13151320 (1988)

11 Schwemmle, M., et al. Unexpected structural requirements for GTPase activity of the interferon-induced MxA protein. J Biol. Chem. 270, 13518-13523 (1995)

12 Zurcher, T., Pavlovic, J., and Staeheli, P. Mechanism of human MxA protein action: variants with changed antiviral properties. EMBO J 11, 1657-1661 (1992)

13 Chappie, J. S., et al. An Intramolecular Signaling Element that Modulates Dynamin Function In Vitro and In Vivo. Mol Biol. Cell 20, 3561-3571 (2009)

14 Prakash, B., et al. Structure of human guanylate-binding protein 1 representing a unique class of GTP-binding proteins. Nature 403, 567-571 (2000)

15 Daumke, O., et al. Architectural and mechanistic insights into an EHD ATPase involved in membrane remodelling. Nature 449, 923-927 (2007)

16 Low, H. H. and Lowe, J. A bacterial dynamin-like protein. Nature 444, 766-769 (2006) 
17 Hinshaw, J. E. and Schmid, S. L. Dynamin self-assembles into rings suggesting a mechanism for coated vesicle budding. Nature 374, 190-192 (1995)

18 Ramachandran, R., et al. The dynamin middle domain is critical for tetramerization and higher-order self-assembly. EMBO J 26, 559-566 (2007)

19 Ingerman, E., et al. Dnm1 forms spirals that are structurally tailored to fit mitochondria. $J$ Cell Biol. 170, 1021-1027 (2005)

20 Kochs, G., et al. Self-assembly of human MxA GTPase into highly ordered dynamin-like oligomers. J Biol. Chem. 277, 14172-14176 (2002)

21 Ponten, A., et al. Dominant-negative mutants of human MxA protein: domains in the carboxy-terminal moiety are important for oligomerization and antiviral activity. J Virol. 71, 2591-2599 (1997)

22 Accola, M. A., et al. The antiviral dynamin family member, MxA, tubulates lipids and localizes to the smooth endoplasmic reticulum. J. Biol. Chem. 277, 21829-21835 (2002)

23 Kochs, G., et al. Assay and functional analysis of dynamin-like Mx proteins. Methods Enzymol. 404, 632-643 (2005)

24 Richter, M. F., et al. Interferon-induced MxA protein. GTP binding and GTP hydrolysis properties. J. Biol. Chem. 270, 13512-13517 (1995)

Melen, K., et al. Enzymatic characterization of interferon-induced antiviral GTPases murine Mx1 and human MxA proteins. J Biol. Chem. 269, 2009-2015 (1994)

Hefti, H. P., et al. Human MxA protein protects mice lacking a functional alpha/beta interferon system against La crosse virus and other lethal viral infections. $J$ Virol. 73, 6984-6991 (1999)

27 Maines, T. R., et al. Avian influenza (H5N1) viruses isolated from humans in Asia in 2004 exhibit increased virulence in mammals. J Virol. 79, 11788-11800 (2005)

28 Dittmann, J., et al. Influenza A virus strains differ in sensitivity to the antiviral action of Mx-GTPase. J Virol. 82, 3624-3631 (2008)

29 Bashkirov, P. V., et al. GTPase cycle of dynamin is coupled to membrane squeeze and release, leading to spontaneous fission. Cell 135, 1276-1286 (2008)

30 Ghosh, A., et al. How guanylate-binding proteins achieve assembly-stimulated processive cleavage of GTP to GMP. Nature 440, 101-104 (2006) 


\section{Figure 1: Structure and oligomerization of the MxA stalk}

a) Schematic representation of the domain structure of human MxA and human Dynamin1. $\alpha$-helices in the MxA stalk are indicated with colours as in Fig. $1 \mathrm{~b}$. Regions not resolved in our structure are indicated by dashed lines.

b) Ribbon-type representation of the MxA stalk with $\mathrm{N}$ - and C-termini labelled. The putative positions of G-domain and substrate of MxA are indicated. Disordered loops are shown as dashed lines.

c) Ribbon-type representation of six oligomerised MxA stalks. The parallel noncrystallographic pseudo two-fold axes across interface-1 and interface-2 are indicated by black dashed lines.

d) Bottom view on interface- 1 between monomer- 4 and 5 with selected residues in the interface shown as ball-and-sticks. Also monomer-2 and 3 in Fig. 1c are associating via this interface. The position of the pseudo two-fold axis is indicated by a filled ellipse.

e) Top view on interface- 2 between monomer-3 and 4 with selected residues in the interface shown as ball-and-sticks. Also monomer-1 and 2 and monomer-5 and 6 in Fig. 1c have this interface in common. The position of the pseudo twofold axis is indicated by a filled ellipse.

f) Bottom view on interface- 3 and $L 4$ with selected residues shown as ball-andsticks. Interface-3 mediates lateral contacts, here between monomer-4 and 6 and also between monomer- 1 and 3, monomer-3 and 5 and monomer-2 and 4 in Fig.1c. Contacts via L4 might stabilise opposing stalks, here between monomer-3 and 6, and monomer-1 and 4 in Fig. 1c. 
Figure 2: Biochemical analysis of the oligomerization interfaces

a) Sedimentation equilibrium experiments were used to determine apparent molecular weights for full-length wt MxA $(\square)$ and the mutants L617D $(\bigcirc)$, $\operatorname{M527D}(\diamond), \operatorname{R408D}(\nabla)$ and $\Delta \mathrm{L} 4(\triangle)$ in dependency of the protein concentration, in the absence of nucleotide at $400 \mathrm{mM} \mathrm{NaCl}$. Data for M527D and L617D were fitted to a monomer-dimer equilibrium equation with a $K_{d}$ of 55 $\mu \mathrm{M} \pm 8 \mu \mathrm{M}$ and $420 \mathrm{nM} \pm 140 \mathrm{nM}$, respectively.

b) Sedimentation experiments for wt MxA and selected mutants in each interface at $2.3 \mathrm{mg} / \mathrm{ml}$ protein concentration were carried out in the absence and presence of $1 \mathrm{mM} \mathrm{GTP}-\gamma-\mathrm{S}$ at $300 \mathrm{mM} \mathrm{NaCl}$. P - Pellet fraction. S Supernatant.

c) TMXA, an artificial nuclear form of MxA carrying the SV40 large T nuclear localization signal and an HA-tag, and the indicated TMXA mutants were coexpressed with FLAG-tagged wt MxA in Vero cells and visualised using antibodies directed against the two tags (Supp. Fig. 7). In co-transfected cells, the fluorescence intensity of FLAG-tagged wt MxA in cytoplasm and nucleus was quantified ( $n=20$ for each experiment).

d) Protein-concentration-dependent GTPase activities of wt MxA $(\square)$ and representative mutants in each interface (L617D $(\bigcirc), \operatorname{M527D}(\diamond), \operatorname{R408D}(\nabla)$ and $\Delta \mathrm{L} 4(\triangle))$ were determined at $150 \mathrm{mM} \mathrm{NaCl}$ and fitted to a quadratic equation. The mean of $\mathrm{k}_{\mathrm{obs}}$ calculated from two independent experiments is indicated with the error bar showing the range of the two data points. 


\section{Figure 3: Antiviral activity of MxA variants}

a) Complex formation of MxA with the LACV nucleoprotein (N). Vero cells transfected with the indicated MxA constructs were infected with LACV for $16 \mathrm{~h}$ and then stained with antibodies specific for MxA (green) and LACV N (red). In the overlays, DAPI staining is shown in blue. $97 \%$ of the wt MxA transfected cells contained MxA/N complexes $(n=100)$ compared to $0 \%$ for all MxA mutants (Supp. Fig. 11). The pictures are representative for three independent experiments (bar=20 $\mu \mathrm{m})$.

b) Minireplicon assay for influenza A virus polymerase. 293T cells were cotransfected with plasmids encoding viral nucleoprotein (NP), the polymerase subunits and a reporter construct encoding fire-fly luciferase under the control of the viral promoter. Expression plasmids for the indicated MxA constructs and for renilla luciferase under a constitutive promoter were co-transfected. $24 \mathrm{~h}$ later, the activity of firefly-luciferase was measured and normalized to the activity of renilla luciferase. The values without MxA expression were set to $100 \%$. Error bars and standard deviations are indicated $(n=3)$. Protein expression was analysed by Western blotting using specific antibodies. 
Figure 4: Oligomeric models of dynamin-like proteins

a, b) Two views on a model of a dynamin oligomer composed of the MxA stalk and the G-domains and PH domains of dynamin as described in Supp. Fig. 12. Stalk dimers assembled via interface- 2 were rotated for $28^{\circ}$ around the indicated axis. Selected positions in interface-3 (Gly392 from L1, Arg408, L2) are indicated.

b) In the front view, the typical T-bar shape of the model becomes obvious. The G-domains of each T-bar structure belong to two neighbouring stalk dimers.

c) Two views on a complete turn of the dynamin helix composed of 13-14 dimers, based on the EM electron density map of oligomerised dynamin in the constricted state. Only after one complete turn is formed, the G-domains of neighbouring helical turns (shown in green-red and blue-yellow surface representations) can approach each other. 


\section{Methods}

Protein Expression and Purification. Human wt MxA and all mutants were expressed as N-terminal His-tag fusions followed by a PreScission cleavage site in Escherichia coli BL21 DE3 Rosetta (Novagen) from a modified pET28 vector. Bacteria cultures in TB medium were induced at an optical density of 0.6 with $45 \mu \mathrm{M}$ IPTG, grown overnight at $19^{\circ} \mathrm{C}$ and lysed in $50 \mathrm{mM}$ HEPES (pH 7.5), $400 \mathrm{mM} \mathrm{NaCl}, 30 \mathrm{mM}$ imidazole, $6 \mathrm{mM} \mathrm{MgCl} 2,1 \mathrm{mM}$ DNase, $2.5 \mathrm{mM} \beta$-Mercaptoethanol ( $\beta$-ME), $500 \mu \mathrm{M}$ Pefabloc SC (Roth) using a microfluidiser (Microfluidics). After centrifugation at $40,000 \mathrm{~g}$ for $45 \mathrm{~min}$ at $4^{\circ} \mathrm{C}$, the soluble extract was filtered and applied to a Ni-NTA column (GE-Healthcare) equilibrated with 50 mM HEPES (pH 7.5), $400 \mathrm{mM} \mathrm{NaCl}, 30$ $\mathrm{mM}$ imidazole, $5 \mathrm{mM} \mathrm{MgCl} 2,2.5 \mathrm{mM} \beta$-ME. The column was extensively washed with $20 \mathrm{mM}$ HEPES (pH 7.5), $800 \mathrm{mM} \mathrm{NaCl}, 5 \mathrm{mM} \mathrm{MgCl} 2,30 \mathrm{mM}$ imidazole, $2.5 \mathrm{mM} \beta$ ME, $1 \mathrm{mM}$ ATP, $10 \mathrm{mM} \mathrm{KCl}$ and shortly with $20 \mathrm{mM}$ HEPES (pH 7.5), $400 \mathrm{mM} \mathrm{NaCl}$, $5 \mathrm{mM} \mathrm{MgCl}_{2}, 80 \mathrm{mM}$ imidazole, $2.5 \mathrm{mM} \beta$-ME. Bound MxA was eluted with $20 \mathrm{mM}$ HEPES (pH 7.5), $400 \mathrm{mM} \mathrm{NaCl}, 300 \mathrm{mM}$ imidazole, $5 \mathrm{mM} \mathrm{MgCl}_{2}, 2.5 \mathrm{mM} \beta-\mathrm{ME}$, and dialysed overnight at $4^{\circ} \mathrm{C}$ against $20 \mathrm{mM}$ HEPES (pH 7.5), $400 \mathrm{mM} \mathrm{NaCl}, 2 \mathrm{mM}$ $\mathrm{MgCl}_{2}, 2.5 \mathrm{mM} \beta$-ME in the presence of $250 \mu \mathrm{g}$ PreScission protease to cleave the $\mathrm{N}$ terminal His-tag. The protein was re-applied to a Ni-NTA column to which it bound under these buffer conditions. Subsequently, the protein was eluted with $20 \mathrm{mM}$ HEPES (pH 7.5), $400 \mathrm{mM} \mathrm{NaCl}, 30 \mathrm{mM}$ imidazole, $2 \mathrm{mM} \mathrm{MgCl} 2,2.5 \mathrm{mM} \beta-\mathrm{ME}$, and PreScission protease was removed via a GST column. MxA was further purified using size-exclusion chromatography on a Superdex200 16/60 column (GE) equilibrated with $20 \mathrm{mM}$ HEPES (pH 7.5), $400 \mathrm{mM} \mathrm{NaCl}, 2 \mathrm{mM} \mathrm{MgCl} 2,2.5 \mathrm{mM}$ dithiothreitol (DTT) where it eluted in a discrete peak at approximately $300 \mathrm{kD}$. Typical yields were $1.5 \mathrm{mg}$ 
purified human MxA protein per $1 \mathrm{~L}$ bacteria culture. Selenomethionine-substituted (SeMet) protein was prepared according to ref. ${ }^{31}$ and purified in the same way as the native protein.

Crystallization and structure determination. Crystallization trials by the sitting-drop vapour-diffusion method were performed at $27^{\circ} \mathrm{C} .400 \mathrm{nl} \mathrm{MxA}(25 \mathrm{mg} / \mathrm{ml})$ were mixed with an equal volume of reservoir solution containing 5\% PEG3350, $100 \mathrm{mM} \mathrm{MES} \mathrm{(pH}$ 6.8), $100 \mathrm{mM} \mathrm{MgCl}_{2}$ and $0.01 \mathrm{mM}$ Hexammine cobalt (III) chloride. Crystals of the native protein appeared after 2 weeks and had dimension of $0.1 \mathrm{~mm} \times 0.05 \mathrm{~mm} \times$ $0.03 \mathrm{~mm}$. Crystals of SeMet protein were obtained in 5\% PEG 3350, $100 \mathrm{mM}$ HEPES ( $\mathrm{pH}$ 7.4), $100 \mathrm{mM} \mathrm{MgCl}$. During flash-cooling of the crystals in liquid nitrogen, a cryo-solution containing 2.5\% PEG3350, 60 mM HEPES (pH 7.4), $50 \mathrm{mM} \mathrm{MgCl} 2,1$ mM DTT, 25\% PEG200 was used for native human MxA crystals and 2.5\% PEG3350, $60 \mathrm{mM}$ HEPES (pH 7.4), $50 \mathrm{mM} \mathrm{MgCl} 2,1 \mathrm{mM}$ DTT, 25\% glycerol was used for SeMet MxA. One data set at the selenium peak wavelength was collected from a single crystal on beamline MX14.1 at BESSY and processed and scaled using the XDS program suite $^{32}$. The phase problem was solved by the single anomalous dispersion method. 16 out of 18 selenium atoms were found with $\operatorname{SHELXD}^{33}$ using the anomalous signal of the peak data set. Initial phases were calculated and refined using the program SHELXE $^{33}$ with the graphical interface hk12map ${ }^{34}$. In the calculated electron density, the main chain was clearly traceable. An initial model was manually built with COOT $^{35}$, where the positions of the 16 selenium atoms were used to assign the sequence. Since the native data set was non-isomorphous to the SeMet data set, molecular replacement with the initial model was carried out against the native data using MOLREP ${ }^{36}$. Restrained and TLS refinement with 2 TLS groups was carried out with Refmac $5^{37}$. The 
final model contains two molecules $(\mathrm{A}, \mathrm{B})$ in the asymmetric unit and has an excellent geometry with $97 \%$ of all residues in the most favoured region in the Ramachandran plot, as determined by Procheck ${ }^{38}$. Molecule A which is described in this manuscript comprises residues 366-438, 448-531, 573-633 (219 residues in total). Molecule B comprises residues $367-435,451-531,576-636$ ( 211 residues in total). The common residues of both molecules can be superimposed with a root mean square deviation of $0.7 \AA ̊$. Interface areas were calculated using $\mathrm{CNS}^{39}$. Figures were prepared using Molscript ${ }^{40}$ and Raster $3 \mathrm{D}^{41}$. The conservation plot was calculated using the Consurf server $^{42}$ and visualised using ccp $4 \mathrm{mg}^{43}$. The model of the oligomerised stalk was created using Swiss PdbViewer ${ }^{44}$ and manually fitted in the electron density map of oligomerised dynamin ${ }^{5}$ using chimera ${ }^{45}$. The oligomer was extended using superpose and pdbset from ccp4. The figure of the oligomer was generated using Pymol ${ }^{46}$. The hydrophobic surface representation was generated using vasco ${ }^{47}$ and Pymol. Pdb coordinates of four molecules of the proposed dynamin oligomer can be found in the Supplementary Materials.

Analytical ultracentrifugation. Molecular mass studies of wt MxA and all mutants in $20 \mathrm{mM}$ HEPES, $\mathrm{pH}$ 7.5, $400 \mathrm{mM} \mathrm{NaCl}, 2 \mathrm{mM} \mathrm{MgCl} 2,2.5 \mathrm{mM}$ DTT were performed in a XL-A type analytical ultracentrifuge (Beckman, Palo Alto, CA) equipped with UV absorbance optics. Sedimentation equilibrium experiments were carried out using sixchannel cells with $12 \mathrm{~mm}$ optical path length and the capacity to handle three solventsolution pairs of about $70 \mu \mathrm{l}$ liquid. Sedimentation equilibrium was reached after $2 \mathrm{~h}$ of overspeed at 16,000 rpm followed by an equilibrium speed of 12,000 rpm for about $30 \mathrm{~h}$ at $10{ }^{\circ} \mathrm{C}$. For some mutants, overspeed of $20,000 \mathrm{rpm}$ and equilibrium speed of 16,000 rpm was used. The radial absorbance in each compartment was recorded at three 
different wave lengths between 270 and $290 \mathrm{~nm}$ depending on the concentration used in the experiments. Molecular mass determinations employed the global fit of the three radial distributions using our program POLYMOLE ${ }^{48}$ or singularly using POLYMOLA. When proteins adopt a monomer-dimer equilibrium, the molecular mass, $\mathrm{M}$, can be treated approximately as a weight average parameter $\left(\mathrm{M}_{\mathrm{w}}\right)$. This value is a composite of the monomer molecular mass $\left(\mathrm{M}_{\mathrm{m}}\right)$ and that of the dimer $\left(\mathrm{M}_{\mathrm{d}}\right)$ and the partial concentrations of monomers, $\mathrm{c}_{\mathrm{m}}$, and dimers, $\mathrm{c}_{\mathrm{d}}$.

$\mathrm{M}_{\mathrm{w}}=\left(\mathrm{c}_{\mathrm{m}} \times \mathrm{M}_{\mathrm{m}}+\mathrm{c}_{\mathrm{d}} \times \mathrm{M}_{\mathrm{d}}\right) /\left(\mathrm{c}_{\mathrm{m}}+\mathrm{c}_{\mathrm{d}}\right)$

Therefore, the equilibrium constant, $\mathrm{K}_{\mathrm{d}}$, can be derived with $\mathrm{K}_{\mathrm{d}}=\mathrm{c}_{\mathrm{m}}{ }^{2} / \mathrm{c}_{\mathrm{d}}$.

GTP hydrolysis assay. GTPase activities of human MxA and the indicated mutants were determined at $37^{\circ} \mathrm{C}$ in $50 \mathrm{mM}$ HEPES (pH 7.5), $150 \mathrm{mM} \mathrm{NaCl}, 5 \mathrm{mM} \mathrm{KCl}, 5 \mathrm{mM}$ $\mathrm{MgCl}_{2}$, using increasing MxA concentrations. Saturating concentrations of GTP (1-4 $\mathrm{mM}$ ) were employed for each reaction. Reactions were initiated by the addition of protein to the final reaction solution. At different time points, reaction aliquots were 20fold diluted in GTPase buffer and quickly transferred in liquid nitrogen. Nucleotides in the samples were separated via a reversed-phase Hypersil ODS-2 C18 column $(250 \times 4$ $\mathrm{mm}$ ), with $10 \mathrm{mM}$ tetrabutylammonium bromide, $100 \mathrm{mM}$ potassium phosphate $(\mathrm{pH}$ $6.5), 7.5 \%$ acetonitrile as running buffer, where denatured proteins were adsorbed at a C18 guard column. Nucleotides were detected by absorption at $254 \mathrm{~nm}$ and quantified by integration of the corresponding peaks. Rates derived from a linear fit to the initial rate of the reaction ( $<40 \%$ GTP hydrolysed) were plotted against the protein concentrations. To estimate $\mathrm{k}_{\max }$, a simple binding model was fitted to the data which describes the interaction of two GTP-bound MxA monomers inducing GTP hydrolysis, as previously done for GBP $1^{49}$. 
Cells and viruses. Human embryonic kidney cells (293T) and Vero cells were maintained in Dulbecco's modified Eagle medium with 10\% fetal calf serum. The original LACV strain from ref. ${ }^{4}$ was used.

Influenza A virus minireplicon system. cDNAs of the viral polymerase subunits (PA,PB1 and PB2) and the viral nucleoprotein (NP) are derived from influenza A/Vietnam/1203/04 virus. 293T cells in 12 well plates were transfected using Nanofectin (PAA). $10 \mathrm{ng}$ of the three plasmids encoding the subunits of viral RNA polymerase and $100 \mathrm{ng}$ for NP were co-transfected with $50 \mathrm{ng}$ of plasmid pPOLI-LucRT carrying the firefly luciferase reporter gene as described ${ }^{28}$. To measure transfection efficiency, $25 \mathrm{ng}$ of the Renilla luciferase-encoding plasmid pRL-SV40-Rluc (Promega) was co-transfected. For MxA expression, $300 \mathrm{ng}$ of the Mx-encoding plasmids were cotransfected. The negative control lacked the plasmid encoding NP. Cells were lysed 24 $\mathrm{h}$ posttransfection. Firefly and Renilla luciferase activities were determined using the Dual Luciferase assay (Promega, Madison, WI).

Western blot analysis. Cell lysates were analysed by SDS-PAGE and Western blot probed with monoclonal mouse antibody M143 directed against $\mathrm{MxA}^{9}$, monoclonal mouse antibody directed against FLUAV nucleoprotein (Serotec), monoclonal mouse antibody against $\beta$-tubulin (Sigma), and horseradish peroxidase-conjugated secondary antibodies.

Immunofluorescence analysis. Vero cells were prepared and stained for MxA proteins and viral antigens by indirect immunofluorescence as described previously ${ }^{4}$. FLAGtagged MxA was detected with mouse monoclonal anti-FLAG M2 (Sigma), HA-tagged MxA with polyclonal rabbit anti-HA (Sigma), untagged MxA with M143, LACV N protein with a polyclonal rabbit antibody. Alexa fluor 555 and Alexa fluor 488 (Invitrogen)-conjugated donkey secondary antibodies and a Zeiss Axioplan 2 microscope with epifluorescence were used for detection. 


\section{Additional References}

Van Duyne, G. D., et al. Atomic structures of the human immunophilin FKBP-12 complexes with FK506 and rapamycin. J Mol Biol. 229, 105-124 (1993)

Kabsch, W Automatic processing of rotation diffraction data from crystals of initially unknown symmetry and cell constants. J. Appl. Cryst. 26, 795-800 (1993)

Sheldrick, G. M. A short history of SHELX. Acta Crystallogr. A 64, 112-122 (2008)

Pape, T and Schneider, TR HKL2MAP: a graphical user interface for phasing with SHELX programs. J. Appl. Cryst. 37, 843-844 (2004)

Emsley, P. and Cowtan, K. Coot: model-building tools for molecular graphics. Acta Crystallogr. D Biol. Crystallogr. 60, 2126-2132 (2004)

Vagin, A. and Teplyakov, A MOLREP: an automated program for molecular replacement. J. Appl. Cryst. 30, 1022-1025 (1997)

Murshudov, G. N., Vagin, A. A., and Dodson, E. J. Refinement of macromolecular structures by the maximum-likelihood method. Acta Crystallogr. D. Biol. Crystallogr. 53, 240-255 (1997)

Laskowski, R. A., et al. Procheck - a program to check the stereochemical quality of protein structures. J. Appl. Cryst. 26, 283-291 (1993)

Brunger, A. T., et al. Crystallography \& NMR system: A new software suite for macromolecular structure determination. Acta Crystallogr. D Biol. Crystallogr. 54, 905-921 (1998)

Kraulis, P. J. Molscript - a Program to Produce Both Detailed and Schematic Plots of Protein Structures. J. Appl. Cryst. 24, 946-950 (1991)

Merritt, E. A. and Murphy, M. E. Raster3D Version 2.0. A program for photorealistic molecular graphics. Acta Crystallogr. D. Biol. Crystallogr. 50, 869-873 (1994)

Landau, M., et al. ConSurf 2005: the projection of evolutionary conservation scores of residues on protein structures. Nucleic Acids Res. 33, W299-W302 (2005)

Potterton, L., et al. Developments in the CCP4 molecular-graphics project. Acta Crystallogr. D Biol. Crystallogr. 60, 2288-2294 (2004)

Guex, N. and Peitsch, M. C. SWISS-MODEL and the Swiss-PdbViewer: an environment for comparative protein modeling. Electrophoresis 18, 2714-2723 (1997)

Pettersen, E. F., et al. UCSF Chimera--a visualization system for exploratory research and analysis. J Comput. Chem. 25, 1605-1612 (2004)

DeLano, W. L. The PyMol Molecular Graphics System. DeLano Scientific, Palo Alto, CA, USA, www.pymol.org (2002) 
47 Steinkellner, G., et al. VASCo: computation and visualization of annotated protein surface contacts. BMC. Bioinformatics 10, 32 (2009)

48 Behlke, J., Ristau, O., and Schonfeld, H. J. Nucleotide-dependent complex formation between the Escherichia coli chaperonins GroEL and GroES studied under equilibrium conditions. Biochemistry 36, 5149-5156 (1997)

49 Praefcke, G. J., et al. Identification of residues in the human guanylate-binding protein 1 critical for nucleotide binding and cooperative GTP hydrolysis. J Mol Biol. 344, 257-269 (2004) 


\section{Reference List}

1 Haller, O., Stertz, S., and Kochs, G. The Mx GTPase family of interferon-induced antiviral proteins. Microbes. Infect. 9, 1636-1643 (2007)

2 Stertz, S., et al. Interferon-induced, antiviral human MxA protein localizes to a distinct subcompartment of the smooth endoplasmic reticulum. J. Interferon Cytokine Res. 26, 650-660 (2006)

3 Kochs, G. and Haller, O. Interferon-induced human MxA GTPase blocks nuclear import of Thogoto virus nucleocapsids. Proc. Natl Acad. Sci. U. S. A 96, 2082-2086 (1999)

4 Reichelt, M., et al. Missorting of LaCrosse virus nucleocapsid protein by the interferoninduced MxA GTPase involves smooth ER membranes. Traffic. 5, 772-784 (2004)

5 Mears, J. A., Ray, P., and Hinshaw, J. E. A corkscrew model for dynamin constriction. Structure. 15, 1190-1202 (2007)

6 Klockow, B., et al. The dynamin A ring complex: molecular organization and nucleotidedependent conformational changes. EMBO J. 21, 240-250 (2002)

7 Chen, Y. J., et al. The stalk region of dynamin drives the constriction of dynamin tubes. Nat. Struct. Mol. Biol. 11, 574-575 (2004)

8 Reubold, T. F., et al. Crystal structure of the GTPase domain of rat dynamin 1. Proc. Natl Acad. Sci. U. S. A 102, 13093-13098 (2005)

9 Flohr, F., et al. The central interactive region of human MxA GTPase is involved in GTPase activation and interaction with viral target structures. FEBS Lett. 463, 2428 (1999)

10 Arnheiter, H. and Haller, O. Antiviral state against influenza virus neutralized by microinjection of antibodies to interferon-induced Mx proteins. EMBO J 7, 13151320 (1988)

11 Schwemmle, M., et al. Unexpected structural requirements for GTPase activity of the interferon-induced MxA protein. J Biol. Chem. 270, 13518-13523 (1995)

12 Zurcher, T., Pavlovic, J., and Staeheli, P. Mechanism of human MxA protein action: variants with changed antiviral properties. EMBO J 11, 1657-1661 (1992)

13 Chappie, J. S., et al. An Intramolecular Signaling Element that Modulates Dynamin Function In Vitro and In Vivo. Mol Biol. Cell 20, 3561-3571 (2009)

14 Prakash, B., et al. Structure of human guanylate-binding protein 1 representing a unique class of GTP-binding proteins. Nature 403, 567-571 (2000)

15 Daumke, O., et al. Architectural and mechanistic insights into an EHD ATPase involved in membrane remodelling. Nature 449, 923-927 (2007) 
17 Hinshaw, J. E. and Schmid, S. L. Dynamin self-assembles into rings suggesting a mechanism for coated vesicle budding. Nature 374, 190-192 (1995)

18

Kabsch, W Automatic processing of rotation diffraction data from crystals of initially unknown symmetry and cell constants. J. Appl. Cryst. 26, 795-800 (1993) 
Pape, T and Schneider, TR HKL2MAP: a graphical user interface for phasing with SHELX programs. J. Appl. Cryst. 37, 843-844 (2004)

Emsley, P. and Cowtan, K. Coot: model-building tools for molecular graphics. Acta Crystallogr. D Biol. Crystallogr. 60, 2126-2132 (2004)

Vagin, A. and Teplyakov, A MOLREP: an automated program for molecular replacement. J. Appl. Cryst. 30, 1022-1025 (1997)

Murshudov, G. N., Vagin, A. A., and Dodson, E. J. Refinement of macromolecular structures by the maximum-likelihood method. Acta Crystallogr. D. Biol. Crystallogr. 53, 240-255 (1997)

Laskowski, R. A., et al. Procheck - a program to check the stereochemical quality of protein structures. J. Appl. Cryst. 26, 283-291 (1993)

Brunger, A. T., et al. Crystallography \& NMR system: A new software suite for macromolecular structure determination. Acta Crystallogr. D Biol. Crystallogr. 54, 905-921 (1998)

Kraulis, P. J. Molscript - a Program to Produce Both Detailed and Schematic Plots of Protein Structures. J. Appl. Cryst. 24, 946-950 (1991)

41 Merritt, E. A. and Murphy, M. E. Raster3D Version 2.0. A program for photorealistic molecular graphics. Acta Crystallogr. D. Biol. Crystallogr. 50, 869-873 (1994)

Landau, M., et al. ConSurf 2005: the projection of evolutionary conservation scores of residues on protein structures. Nucleic Acids Res. 33, W299-W302 (2005)

Potterton, L., et al. Developments in the CCP4 molecular-graphics project. Acta Crystallogr. D Biol. Crystallogr. 60, 2288-2294 (2004)

Guex, N. and Peitsch, M. C. SWISS-MODEL and the Swiss-PdbViewer: an environment for comparative protein modeling. Electrophoresis 18, 2714-2723 (1997)

Pettersen, E. F., et al. UCSF Chimera--a visualization system for exploratory research and analysis. J Comput. Chem. 25, 1605-1612 (2004)

DeLano, W. L. The PyMol Molecular Graphics System. DeLano Scientific, Palo Alto, CA, USA, www.pymol.org (2002)

47 Steinkellner, G., et al. VASCo: computation and visualization of annotated protein surface contacts. BMC. Bioinformatics 10, 32 (2009)

48 Behlke, J., Ristau, O., and Schonfeld, H. J. Nucleotide-dependent complex formation between the Escherichia coli chaperonins GroEL and GroES studied under equilibrium conditions. Biochemistry 36, 5149-5156 (1997) critical for nucleotide binding and cooperative GTP hydrolysis. J Mol Biol. 344, 257-269 (2004) 


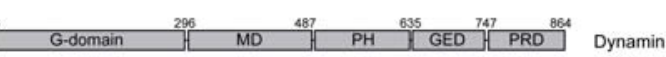

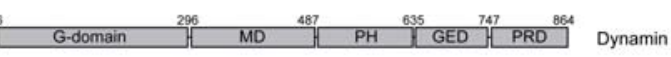

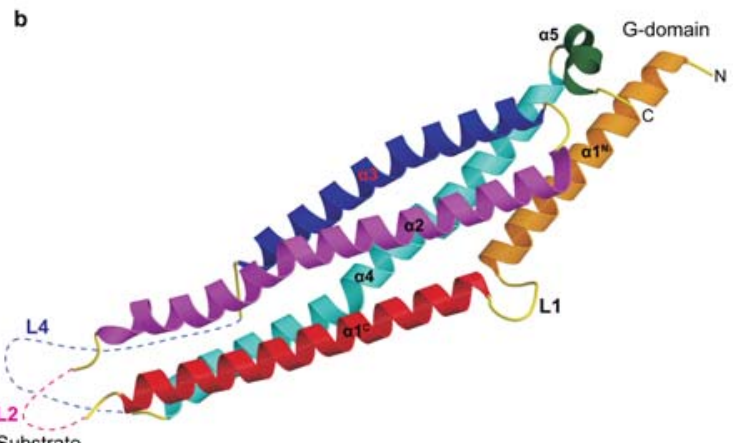

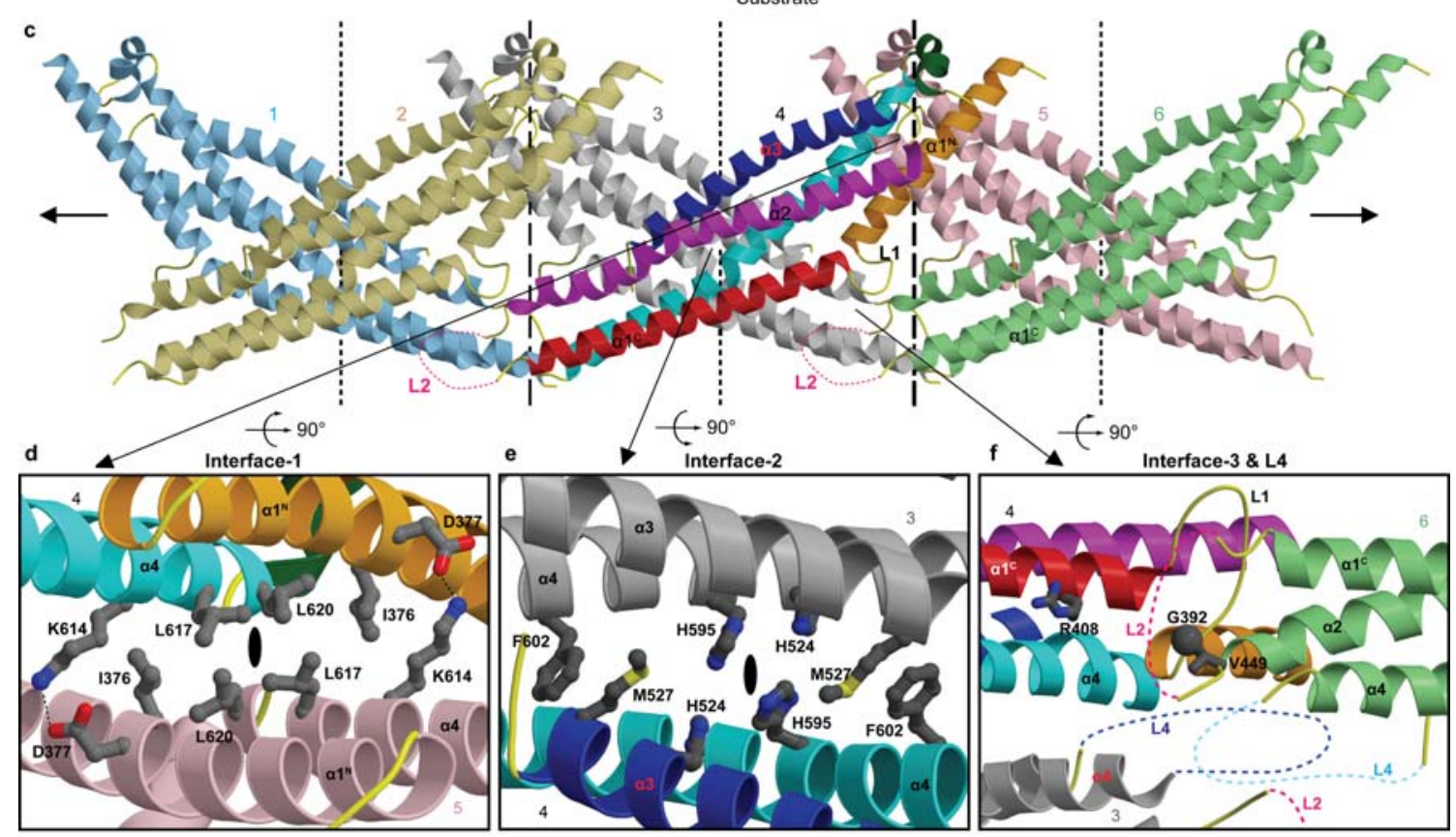

Figure 1 


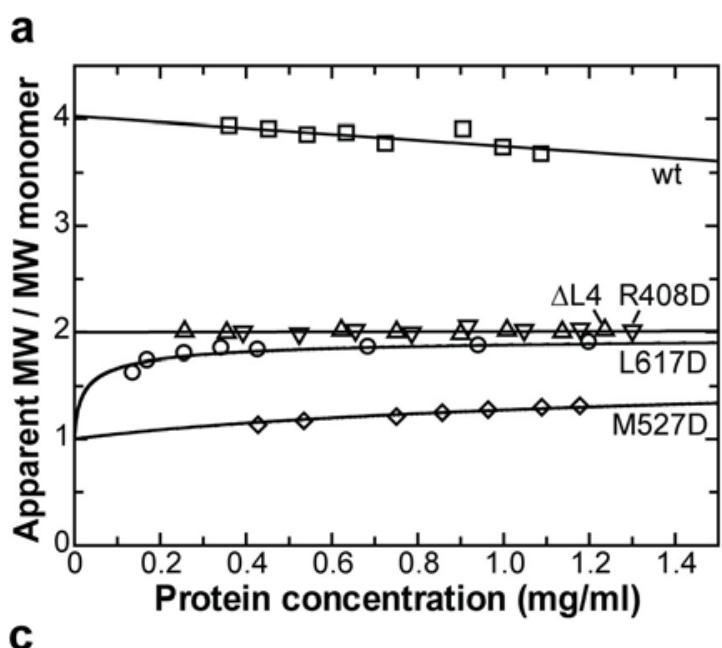

b

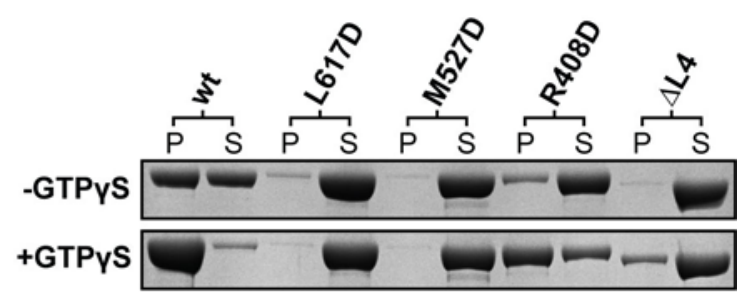

c
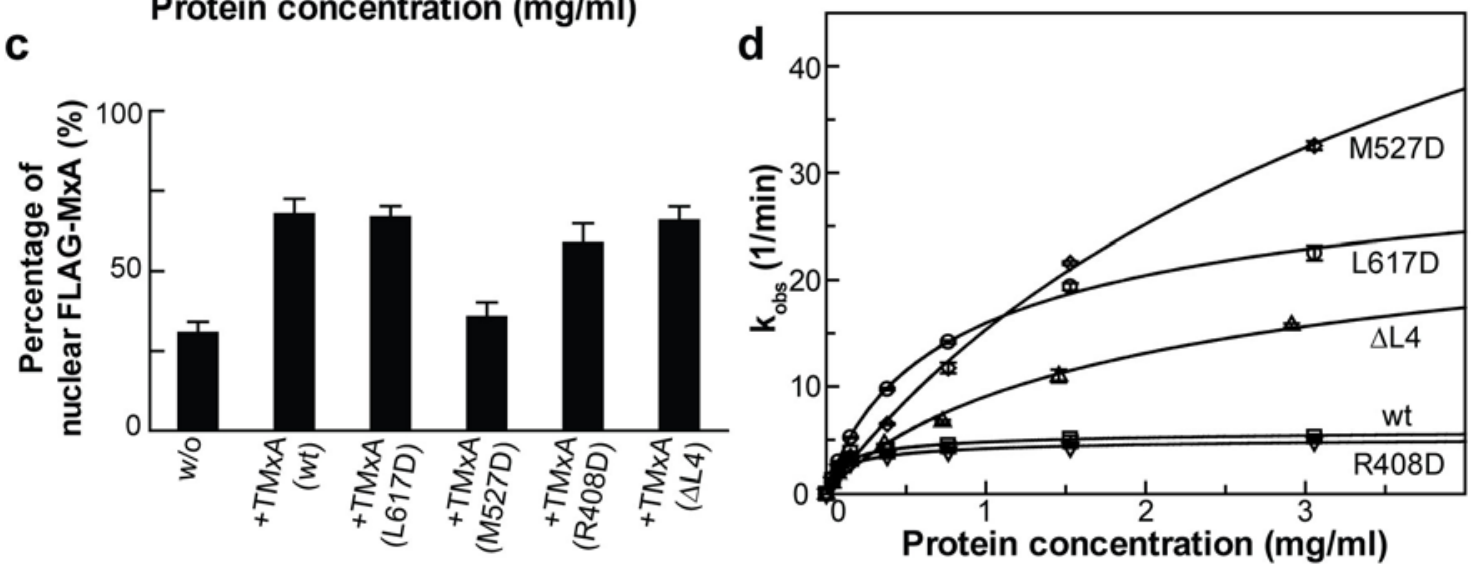

Protein concentration $(\mathrm{mg} / \mathrm{ml})$

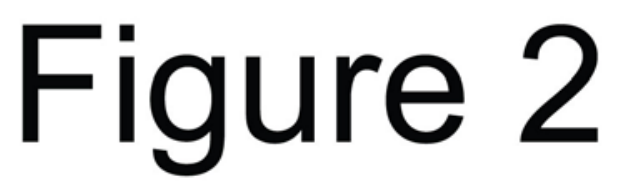




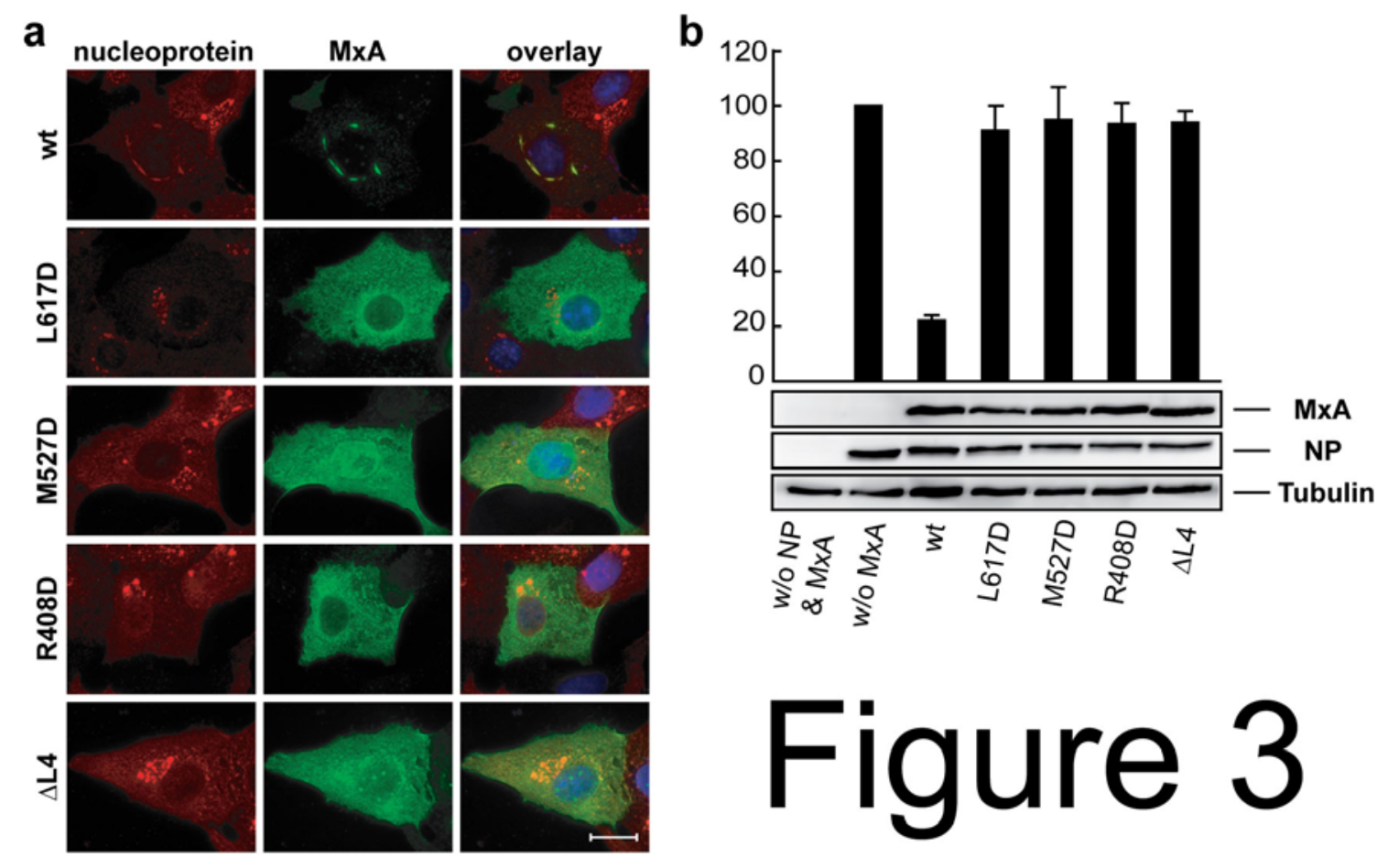




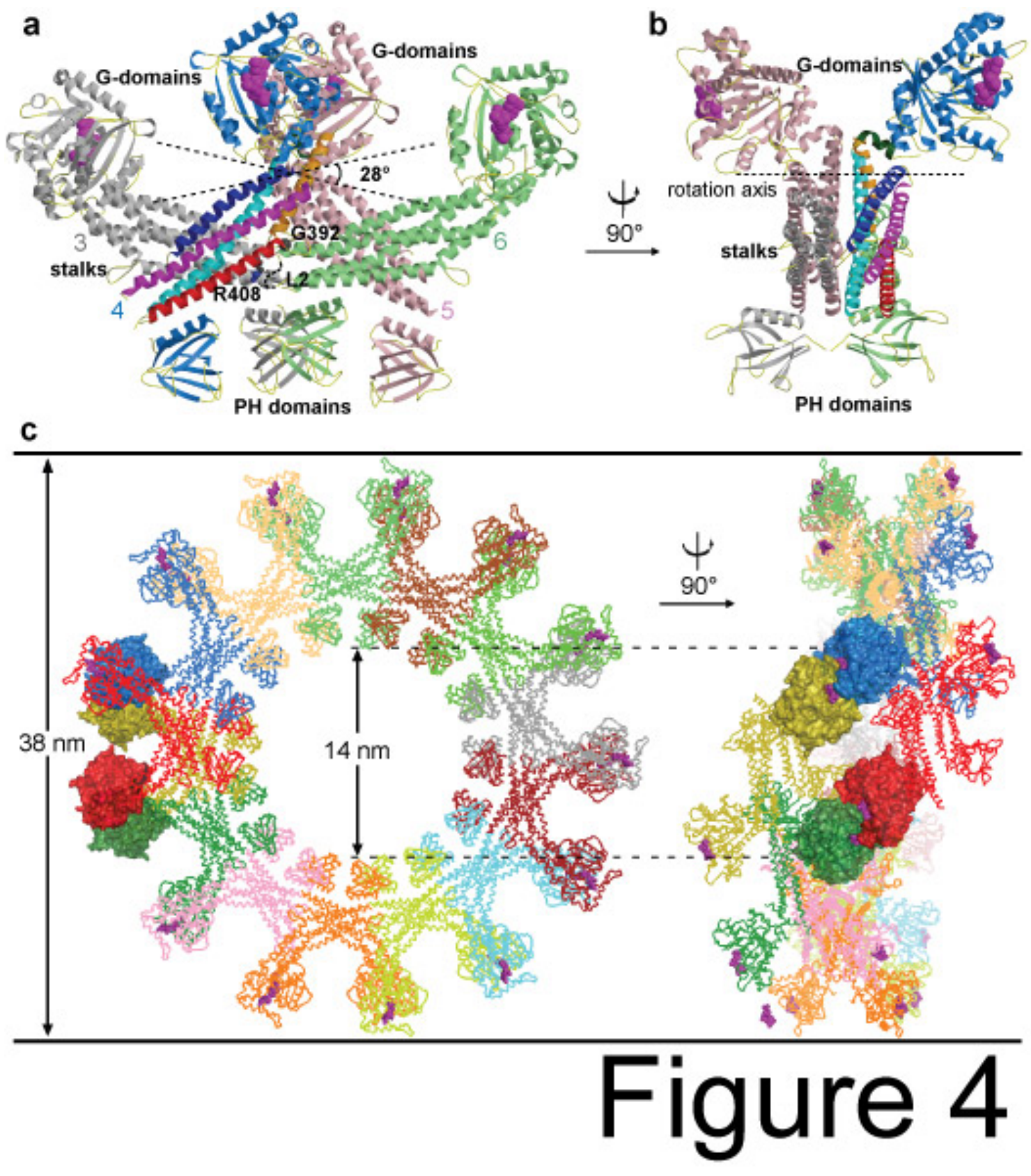




\section{Supplementary Information}

Supplementary Figure 1: Expression and purification of full-length MxA

Supplementary Figure 2: Analysis of dissolved MxA protein needles

Supplementary Figure 3: Alignment of Mx and dynamin proteins

Supplementary Figure 4: Structural comparison of the MxA stalk

Supplementary Figure 5: Sequence conservation within the stalk region

Supplementary Figure 6: Assembly of MxA

Supplementary Figure 7: Nuclear translocation assay

Supplementary Figure 8: Co-immunoprecipitation studies of wt MxA with the indicated MxA mutants

Supplementary Figure 9: Liposome binding of MxA and mutants

Supplementary Figure 10: Nucleotide binding characteristics of MxA

Supplementary Figure 11: Quantification of the $\mathbf{M x A} / \mathbf{N}$ positive complexes

Supplementary Figure 12: Construction of the dynamin oligomer

Supplementary Table 1: Data collection statistics

Supplementary Table 2: $\quad$ Refinement statistics

Supplementary Notes: References of the Supplementary Material 


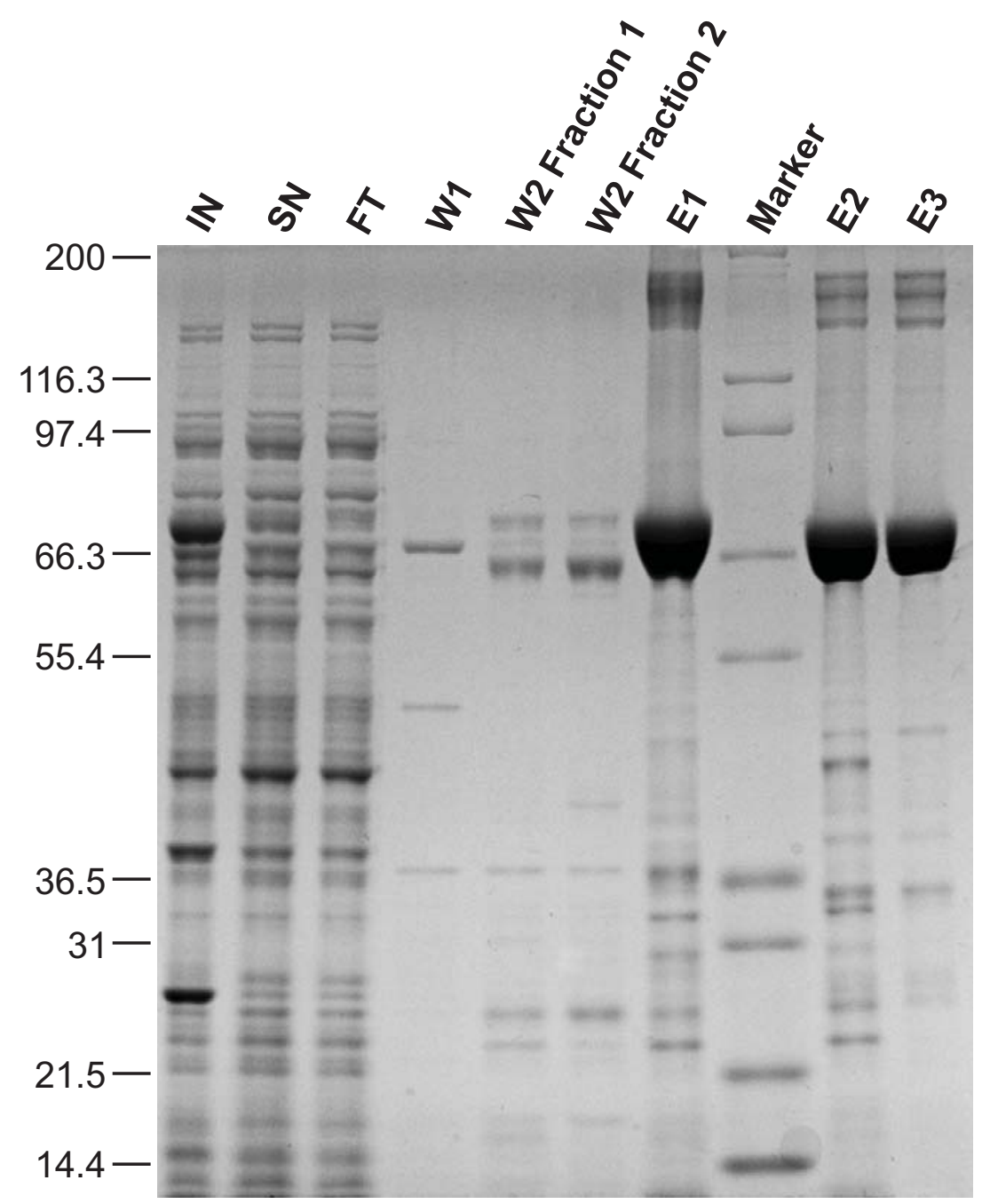

\section{Supplementary Figure 1: Expression and purification of full-length MxA}

Human MxA was expressed in Escherichia coli as a His-tag fusion protein, as described in Methods. IN - Induced culture. SN - Soluble extract. FT - soluble extract after application to Ni-NTA Sepharose. W1 - flow-through of high-salt and ATP wash and W2 (Fraction 1 and 2) - of high-imidazole wash. E1 - MxA after elution from Ni-NTA Sepharose. E2 - MxA after dialysis and PreScission cleavage. E3 - MxA after reapplication and elution from the Ni-NTA column. MxA was further purified using a Superdex200 gel filtration column where it eluted as a distinct peak separated from the exclusion volume (see also Supp. Fig. 6c). 


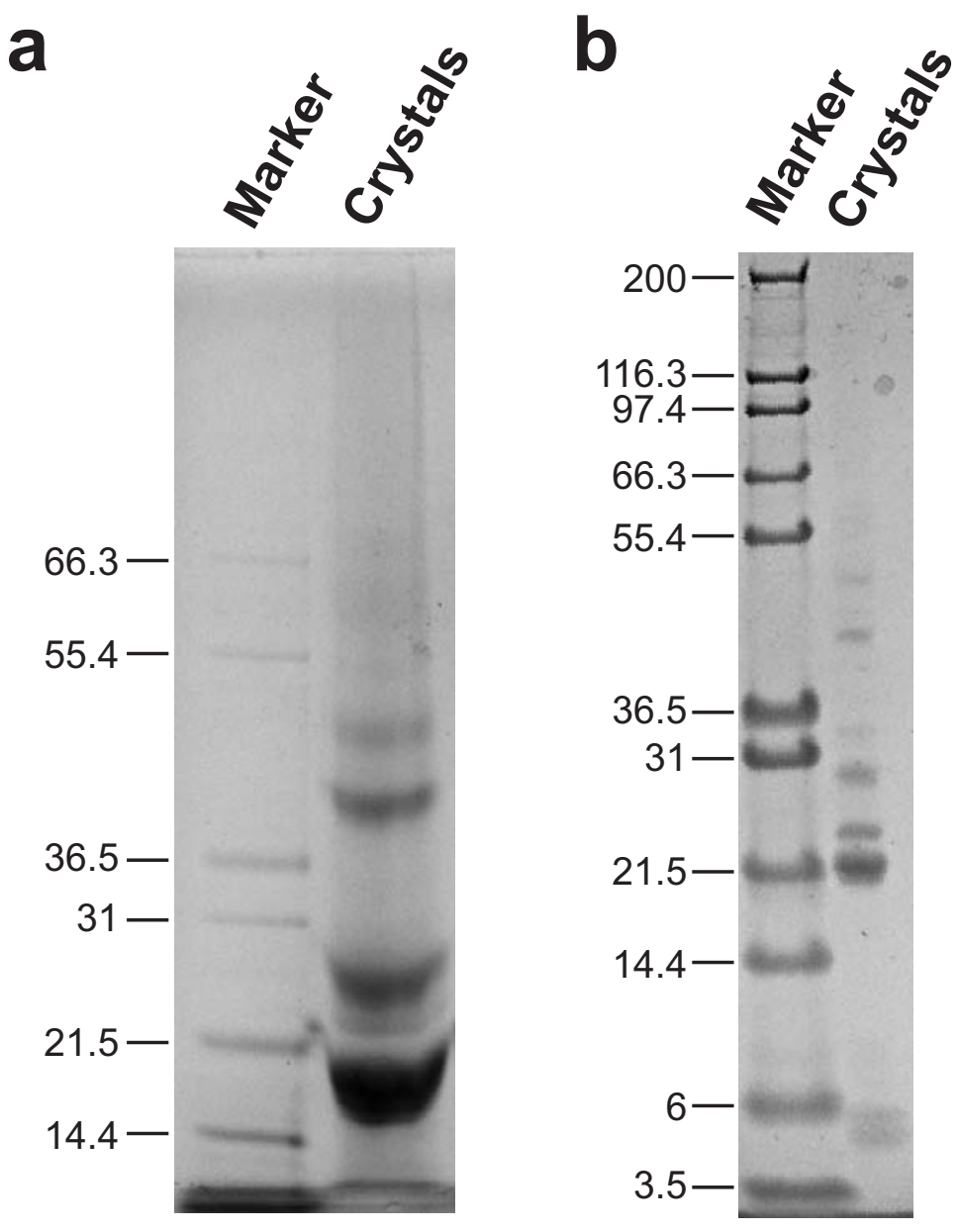

\section{Supplementary Figure 2: Analysis of dissolved MxA protein needles}

Approximately $100 \mathrm{MxA}$ crystals were collected with a small loop, washed in reservoir solution and separated by SDS-PAGE in either MOPS (a) or MES buffer (b). A nonhomogenous mixture of fragments of varying sizes was detected, and all fragments contained peptides of the MD and/or GED of MxA, as determined by in-gel digests and MALDI-TOF analysis. Most of the fragments were smaller than $35 \mathrm{kD}$ (size of the fulllength stalk in our model) indicating that at least one further proteolytic cleavage has occurred in the crystallised fragment. The non-homogeneity of these samples prevented us from determining exact boundaries of each fragment. Consequently, the boundaries of our stalk model were determined solely based on evidence from the electron density. 
hsMxA hsMxB

mmMx 1

$\mathrm{mmMx} 2$

ggMx

drMxA

hsDyn 1

hsDyn2

hsDyn 3

dmDyn

ceDyn

sCDNM1
---------- ---------- --------- ---------- --------MV VSEVDIAKAD 12

MSKAHKPWPY RRRSQFSSRK YLKKEMNSFQ QQPPPFGTVP PQMMFPPNWQ GAEKDAAFLA

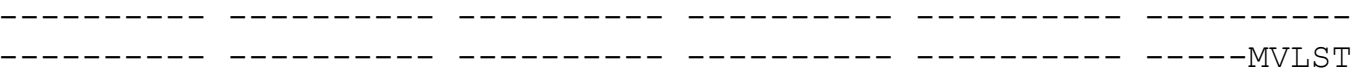

----MNNPWS NFSSAFGCPI QIPKQNSNVP PSLPVPVGVF GVPLRSGCSN QMAFCAPELT
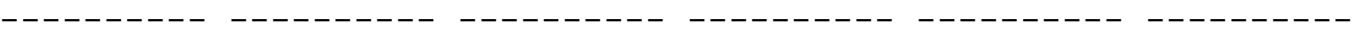

$---------$

hsMXA

hsMxB

$\operatorname{mmMx} 1$

$\mathrm{mmMx} 2$

$\operatorname{ggMx}$

$\operatorname{drMxA}$

hsDyn1

hsDyn2

hsDyn3

dmDyn

ceDyn

sCDNM1

PAAASHPLLL NGDATVAQKN PGSVAENNLC SQYEEKVR KDFNFLTLNN QPPPGNRSQP RAMGPENNLY SQYEQKVR

--------- --------- --MDSVNNLC RHYEEKVR

EENTGVDSVN LPSGETGLGE KDQESVNNLC SQYEEKVR

DRKPEHEQKV SKRLNDREED KDEAAACSLD NQYDRKIQ

---------- ---------- -MEKLSYTFS QQYEEKIRPC

IDLIDSLRAL
IDLIDSLRAL
IDLIDTLRAL
IDLIDSLRAL G

GVEQDLALPA 72

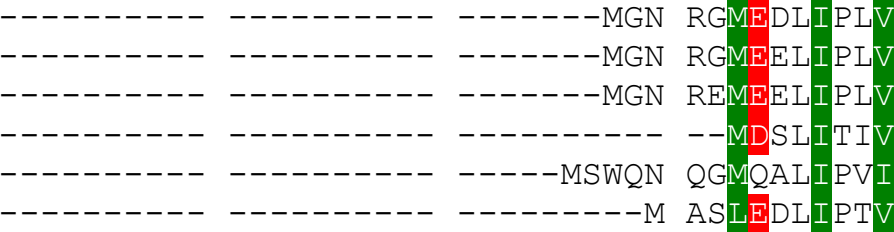

IDLVDSLRKL DIGNDLMLPA 116

IDTIDNLRSL GVEKDLALPA 39

NRLQDAFSAI GQNADLDLPQ 33

NKLQDAFSSI GQSCHLDLPQ 33

NRLQDAFSAL GQSCLLELPQ 33

NKLQDAFTSL GVHMQLDLPQ 28

NRVQDAFSQI GTSVSFELPQ 35

30

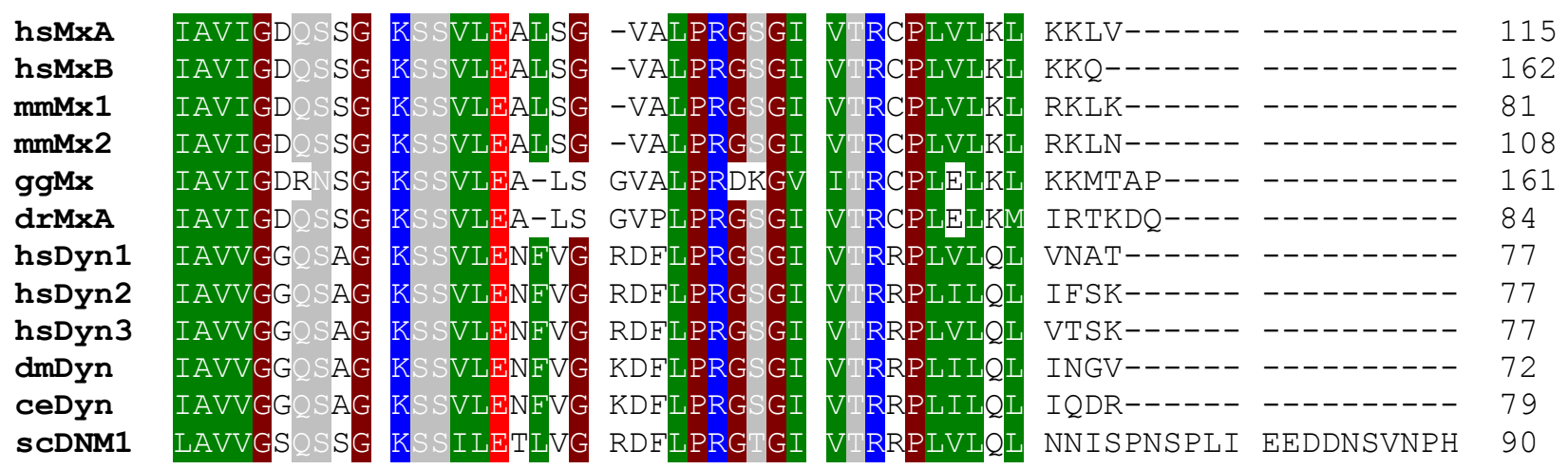

115

162

81

84

77

77

77

72

90

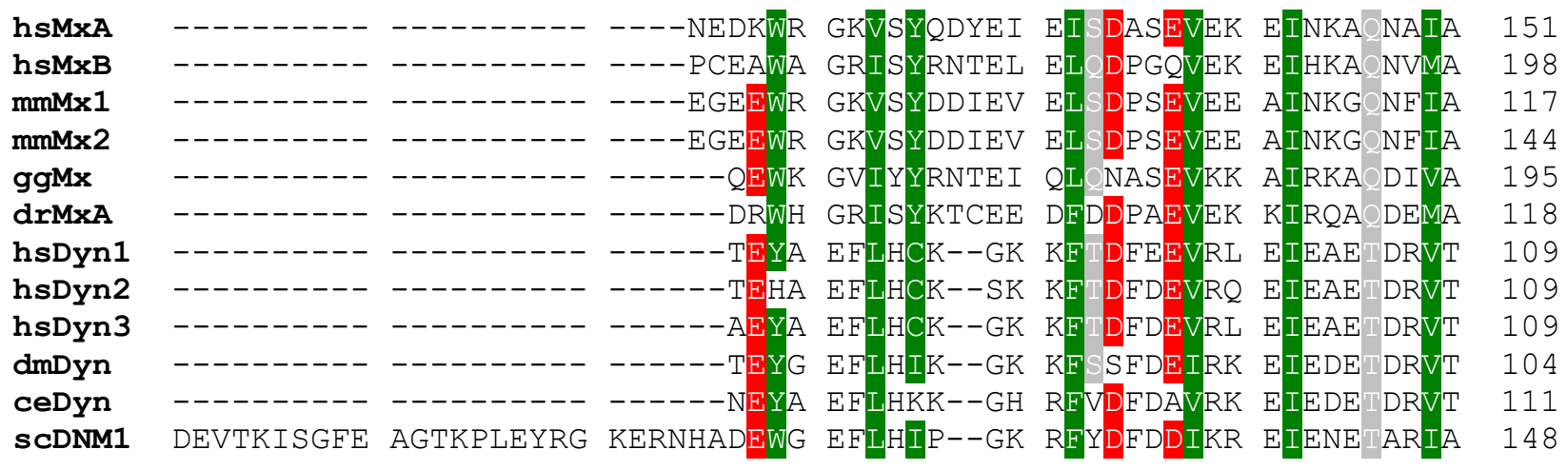

\section{Supplementary Figure 3}



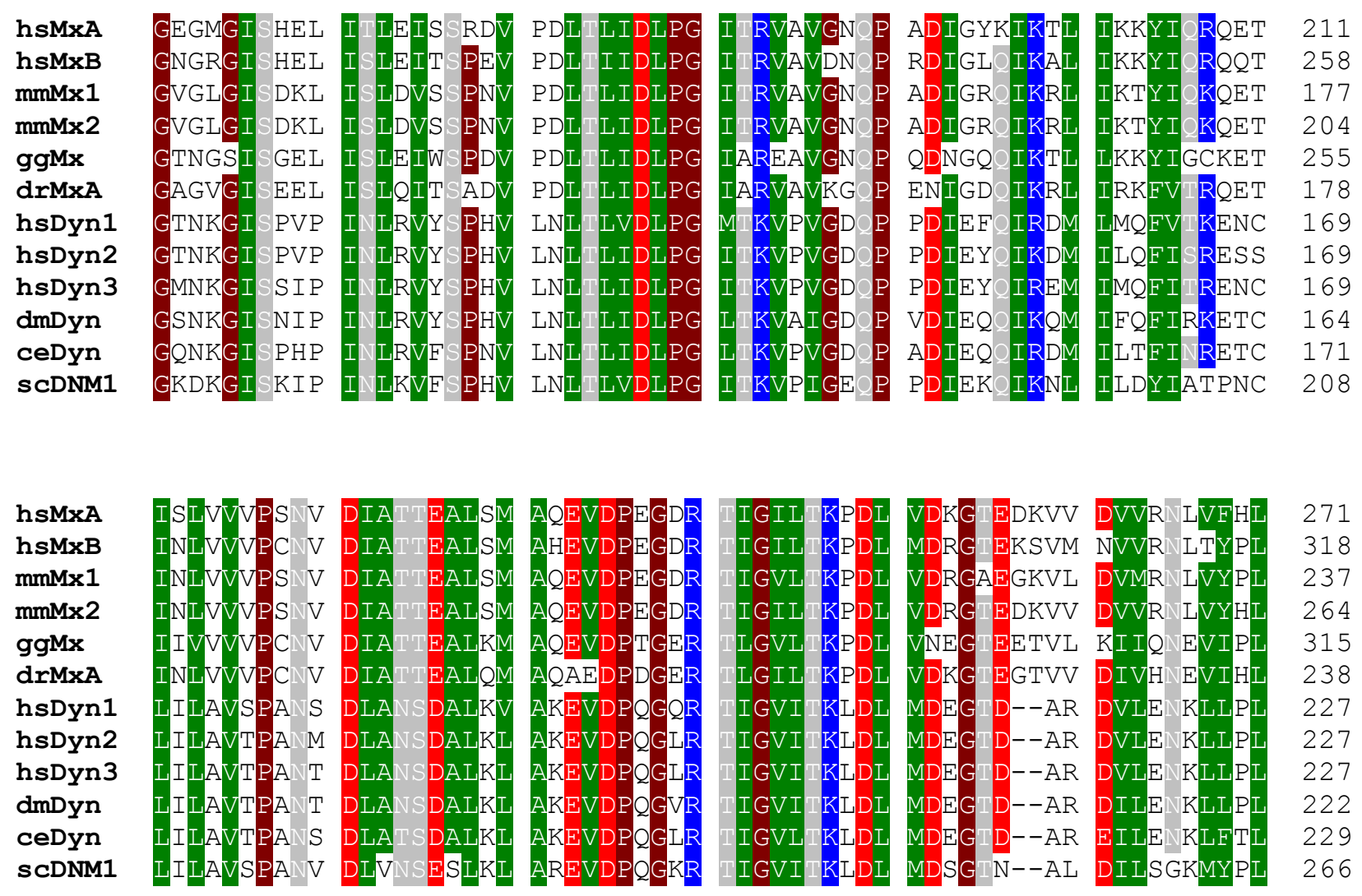

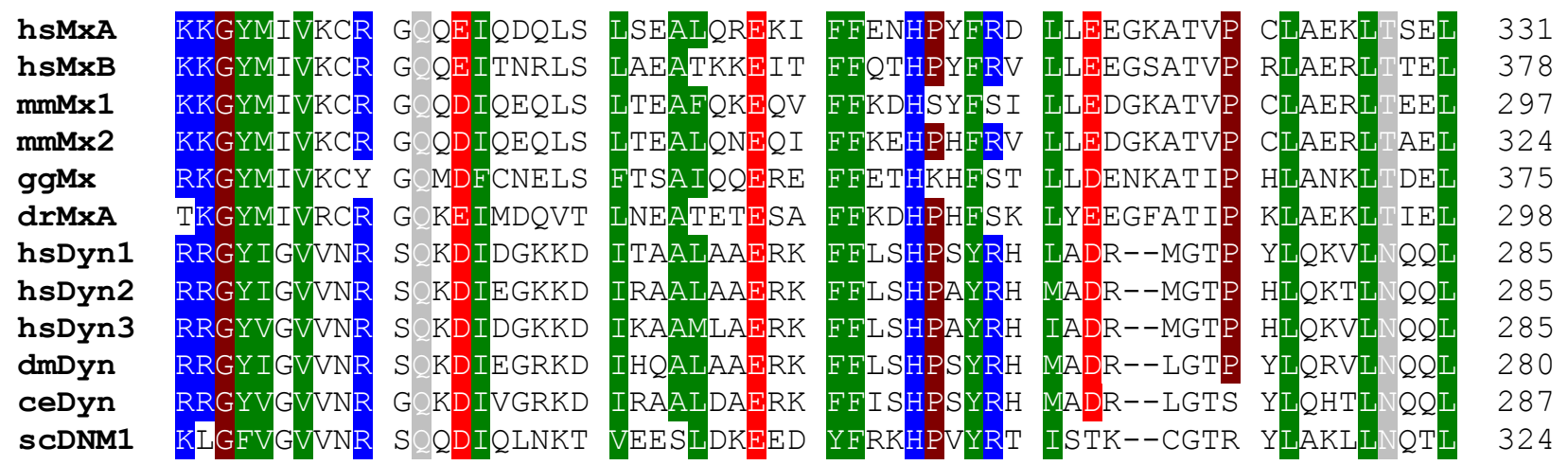

\section{Supplementary Figure 3}



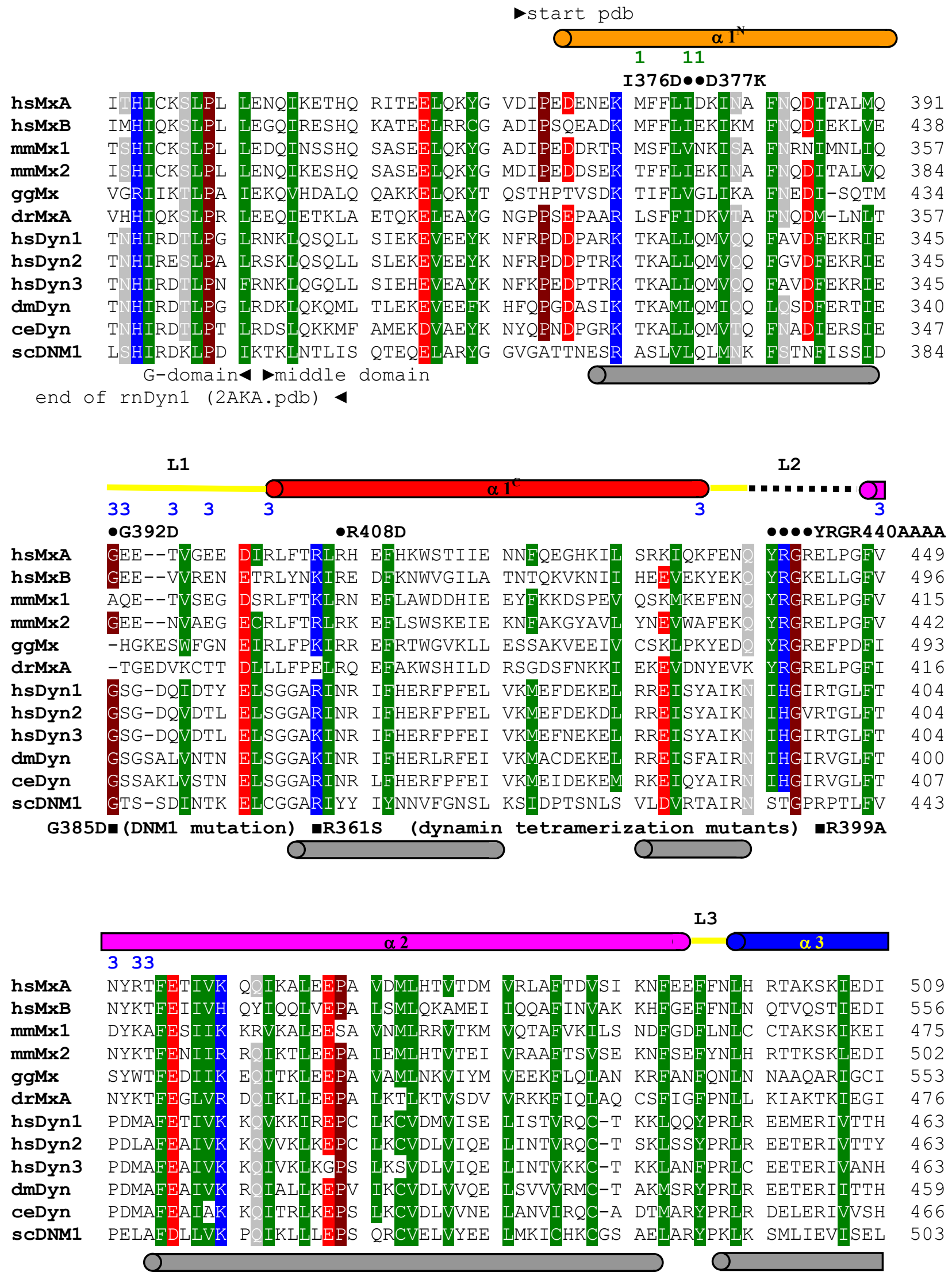

\section{Supplementary Figure 3}




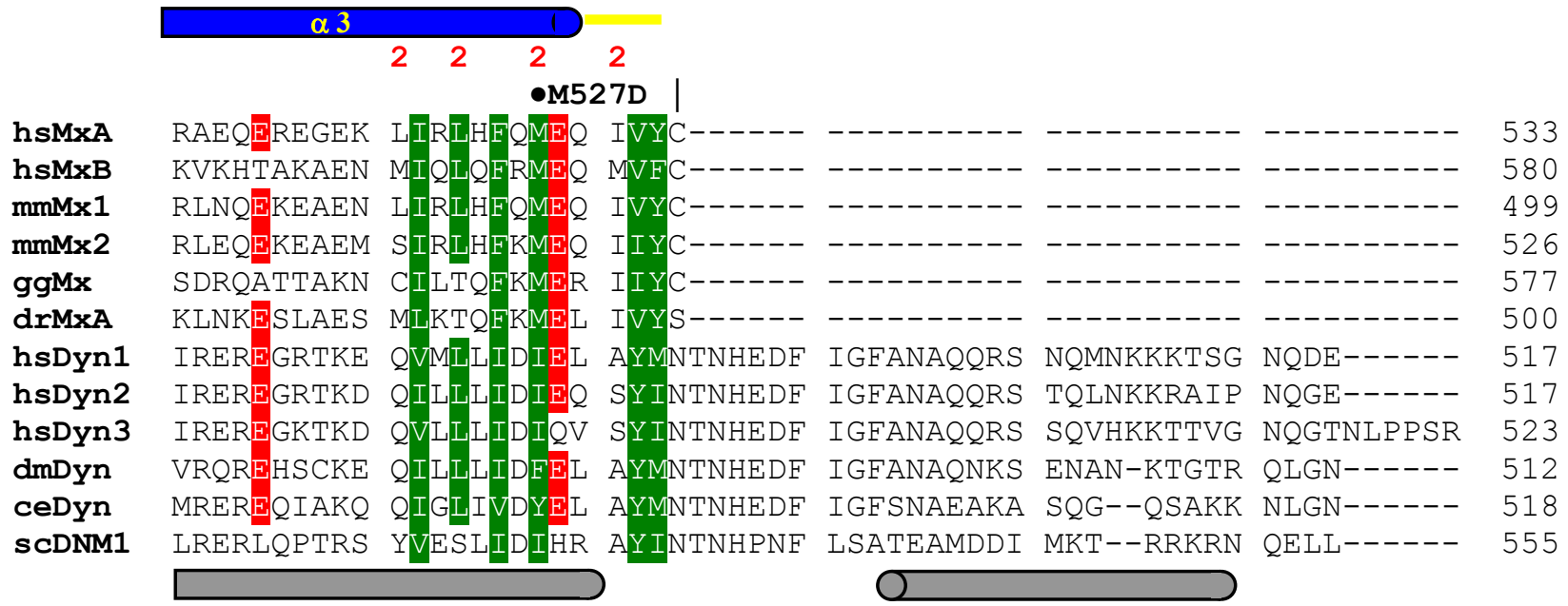

L4

hsMxA
hsMxB
mmMx1
mmMx2
ggMx
drMxA
hsDyn1
hsDyn2
hsDyn3
dmDyn
ceDyn
scDNM1

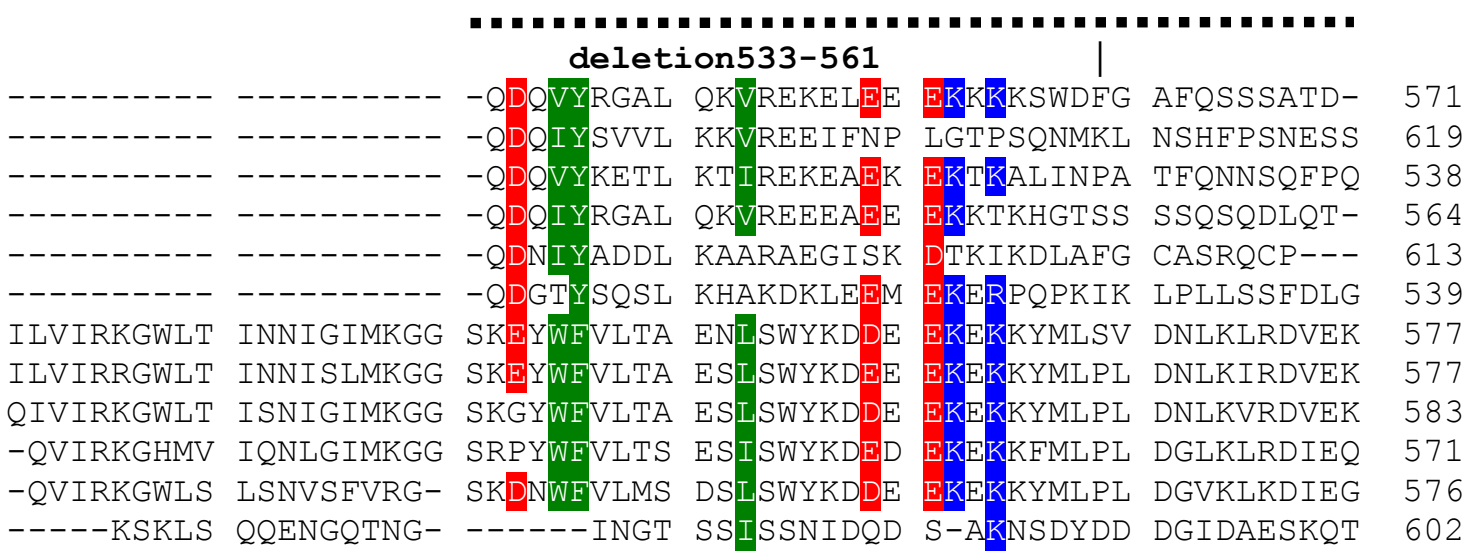

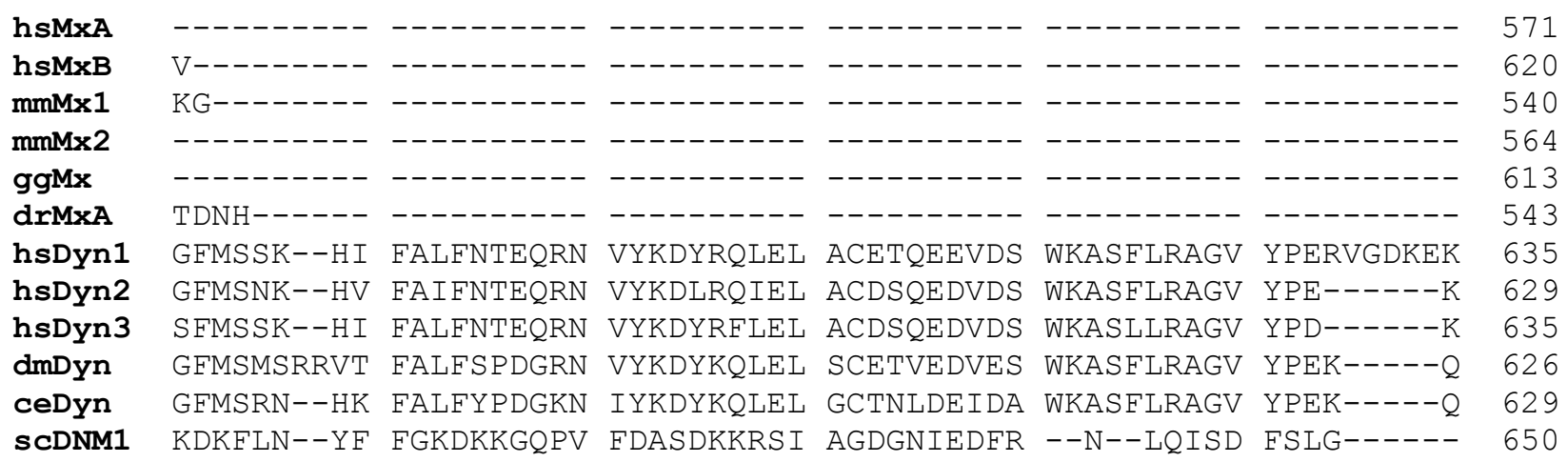

\section{Supplementary Figure 3}


hsMXA

hsMxB

$\mathrm{mmMx} 1$

$\mathrm{mmMx} 2$

ggMx

$\operatorname{drMxA}$

hsDyn1

hsDyn2

hsDyn3

dmDyn

ceDyn

sCDNM1

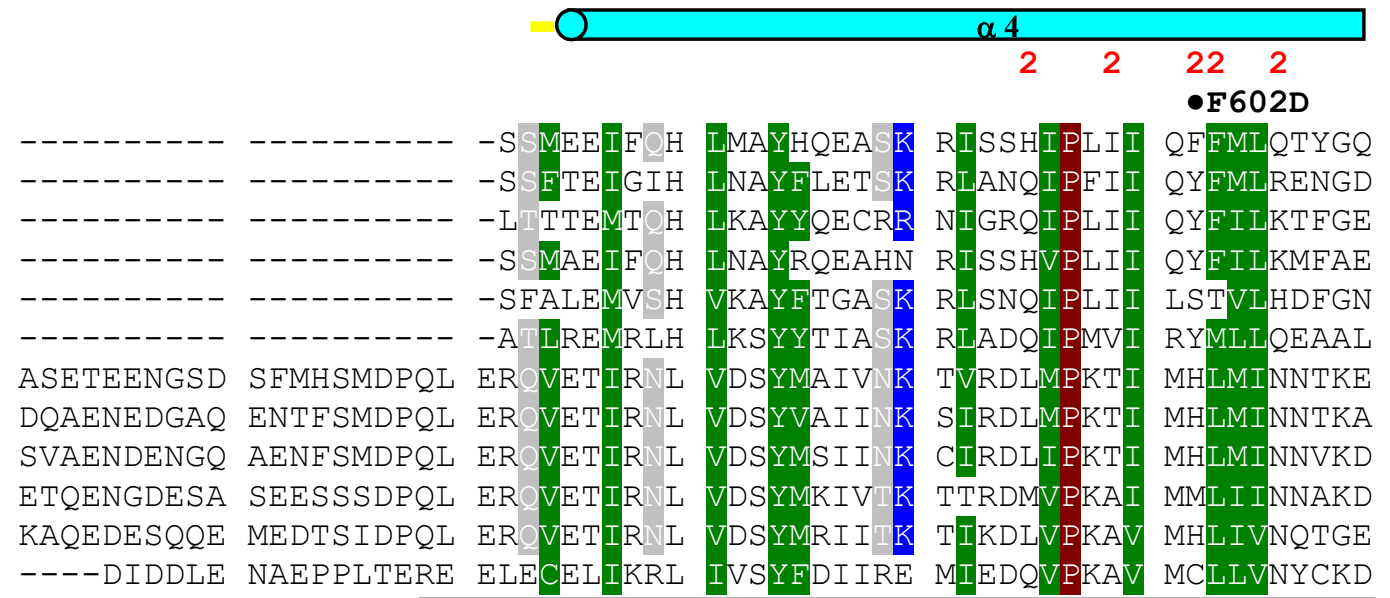

610

659

579

603

652

582

695

689

695

686

689

706

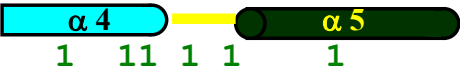 \\ L617D}

K614D• • -L620D

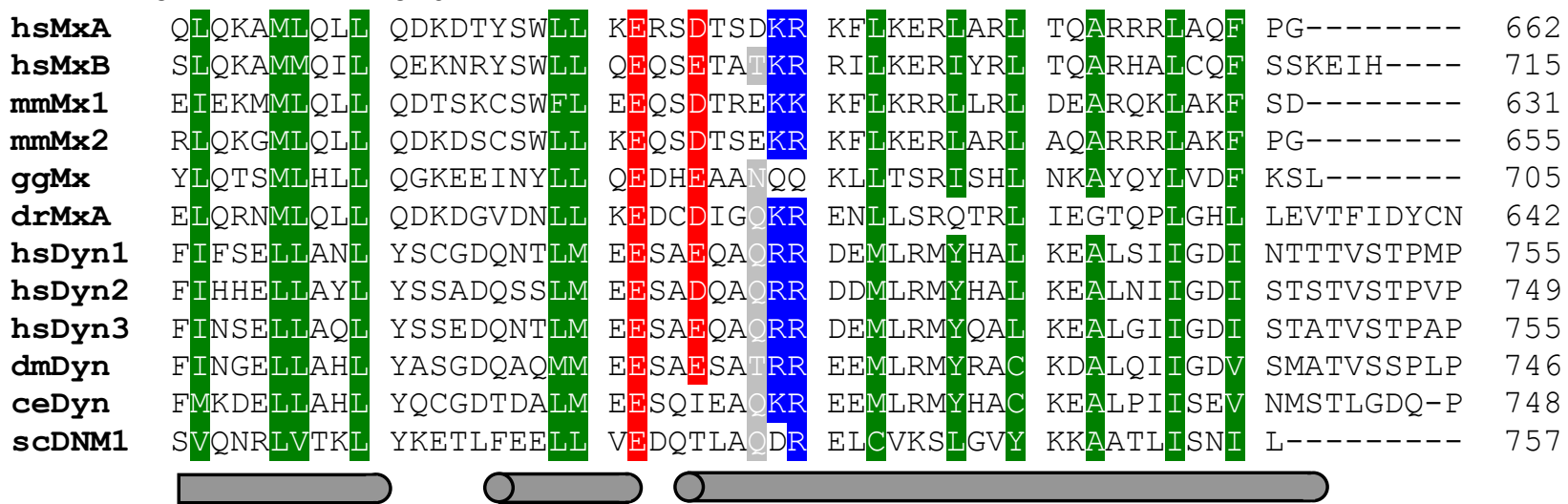

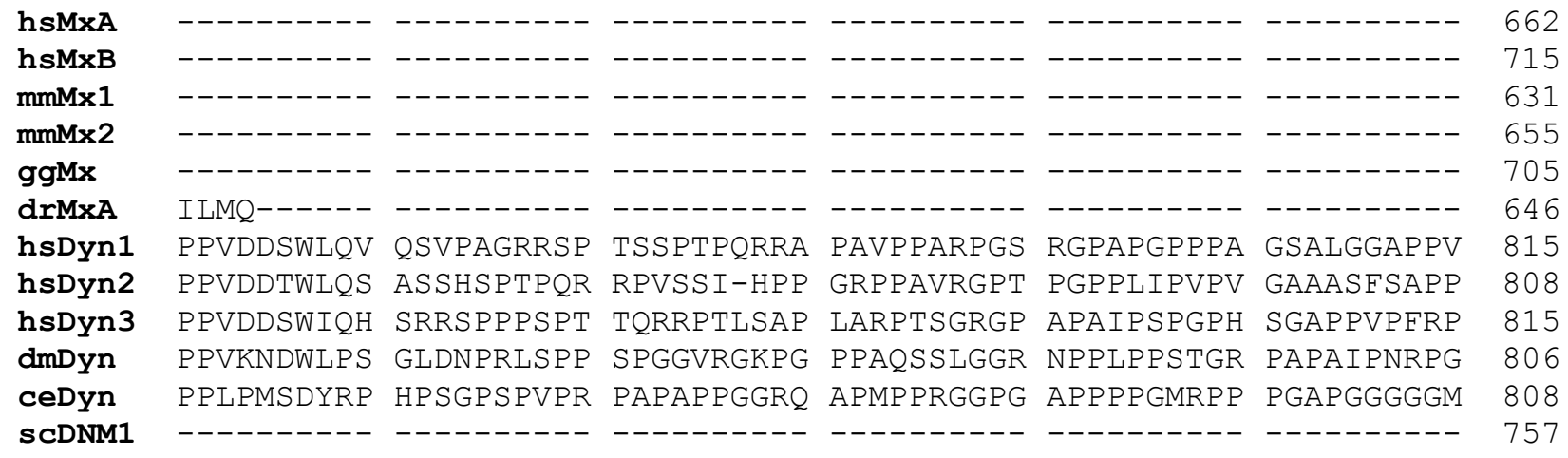

\section{Supplementary Figure 3}




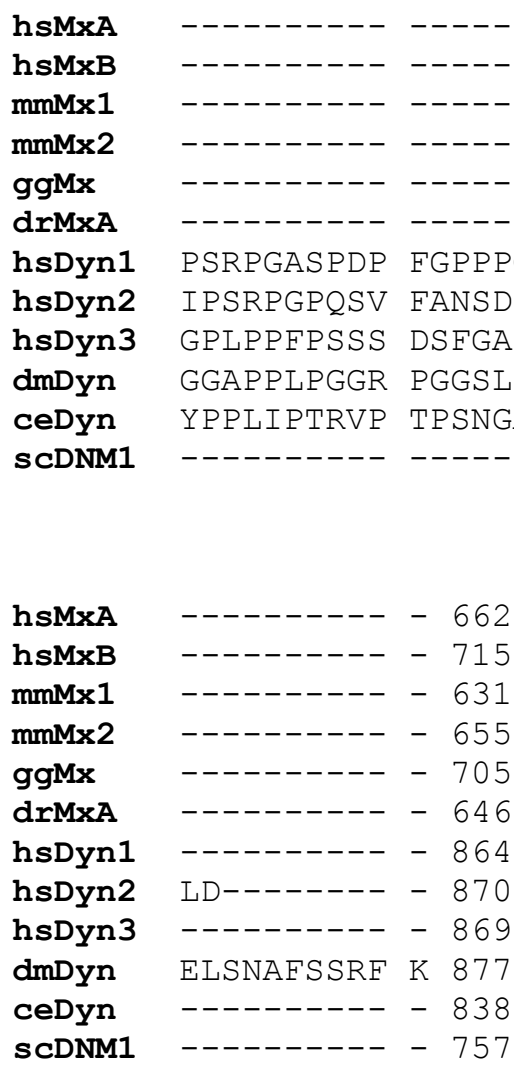

\section{Supplementary Figure 3: Alignment of Mx and dynamin proteins}

Amino acid sequences of human (hs) MxA (Swiss-Prot accession P20591), human MxB (P20592), mouse (mm) Mx1 (P09922), mouse Mx2 (Q9WVP9), chicken (gg) Mx protein (Q90597), zebrafish (dr) MxA protein (Q8JH68), human Dynamin1 (Q05193), human Dynamin2 (P50570), human Dynamin3 (Q9UQ16), Drosophila melanogaster (dm) Dynamin (P27619), Caenorhabditis elegans (ce) Dynamin (Q9U9I9) and Saccharomyces cerevisiae (sc) dynamin-related protein DNM1 (P54861) were aligned using CLUSTAL $\mathrm{W}^{50}$ and manually adjusted. Residues with a conservation of greater than $70 \%$ are colour-coded (D,E in red; R,K,H in blue; N,Q,S,T in grey; L, I, V, F, Y, W, M, C in green). Alphahelices are shown as cylinders with colours as in Fig. 1b. The secondary structure prediction for dynamin (grey helices), as determined by jpred ${ }^{51}$, is in good accordance with this assignment. The PH domain of dynamin is indicated by a green line with domain boundaries taken from the structure of the $\mathrm{PH}$ domain $^{52}$. Residues in the interfaces were identified with Ligplot ${ }^{53}$ and manually confirmed (numbers on top correspond to the interfaces involved). Mx mutants generated in this study are indicated $(\bullet)$ as well as dimerization mutants of dynamin ${ }^{18}$ and $\operatorname{scDNM}^{19,54}(\boldsymbol{\Xi})$ and residues of dynamin involved in $\mathrm{PIP}_{2}$ binding $^{55,56}(\bullet)$. 

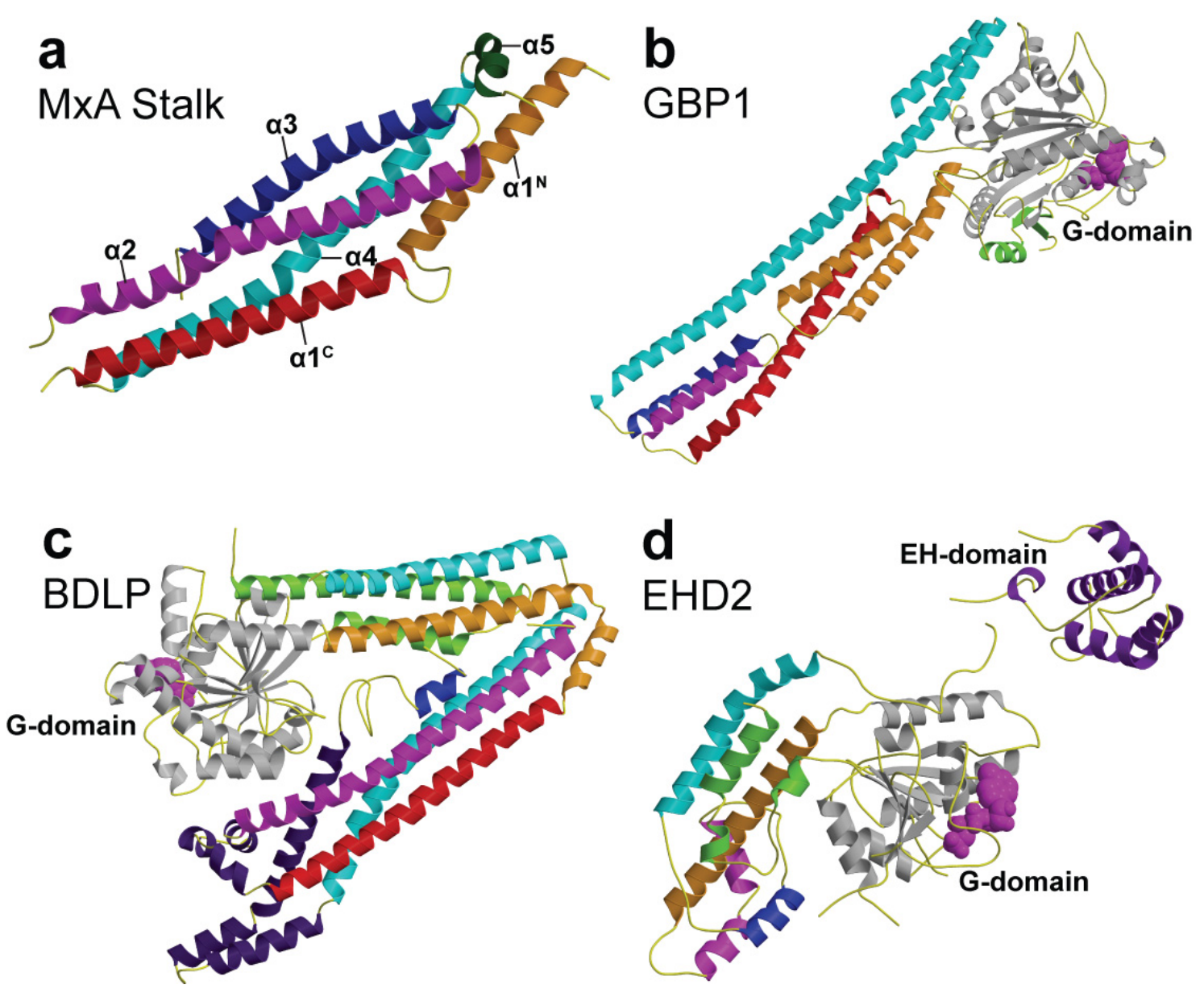

\section{Supplementary Figure 4: Structural comparison of the MxA stalk}

Pdb coordinates of GMP-PNP bound Interferon-induced guanylate-binding protein 1 (GBP1) (b, pdb accession code 1f5n $)^{57}$, GDP-bound bacterial dynamin-like protein (BDLP) (c, 2j68) ${ }^{16}$ and ATP- $\gamma$-S bound EH-domain containing protein 2 (EHD2) (d, 2qpt) ${ }^{15}$ are shown in comparison with the stalk of MxA (a). G-domains of GBP1, BDLP and EHD2 are shown in grey with nucleotides in magenta. Additional elements such as the tip of the paddle in $\mathrm{BDLP}^{16}$ or the EH-domain in EHD2 are shown in violet.

The architecture of the MxA stalk differs from that of other dynamin superfamily members (b-d), although they all have a long N-terminal helix in common leading away from the G-domain (shown in orange and red) and a GED-like helix (cyan) leading back to the G-domain. In BDLP and EHD2, additional helices (green) derived from residues further N-terminal of the G-domain participate in the helical assembly. 
a

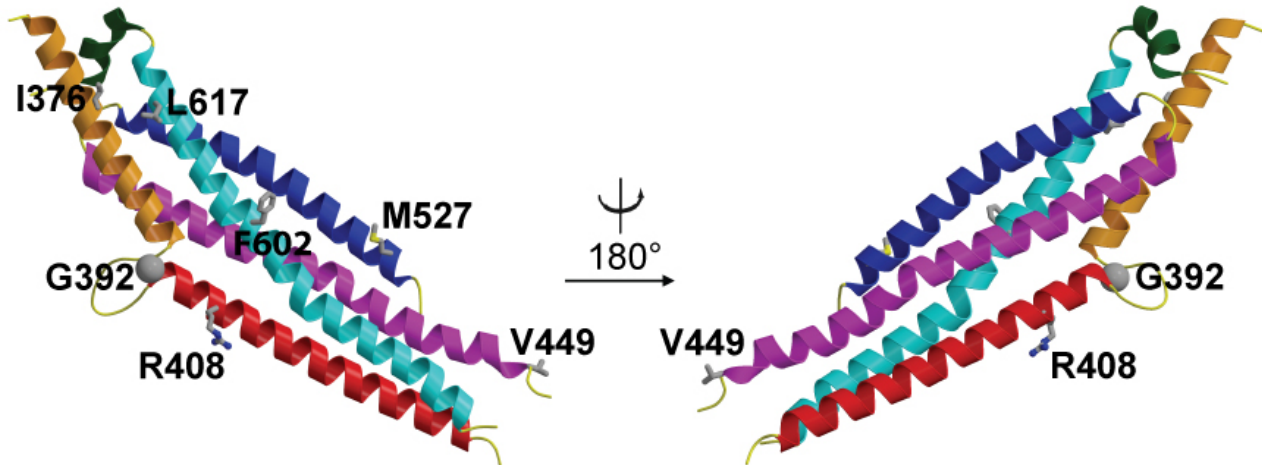

b
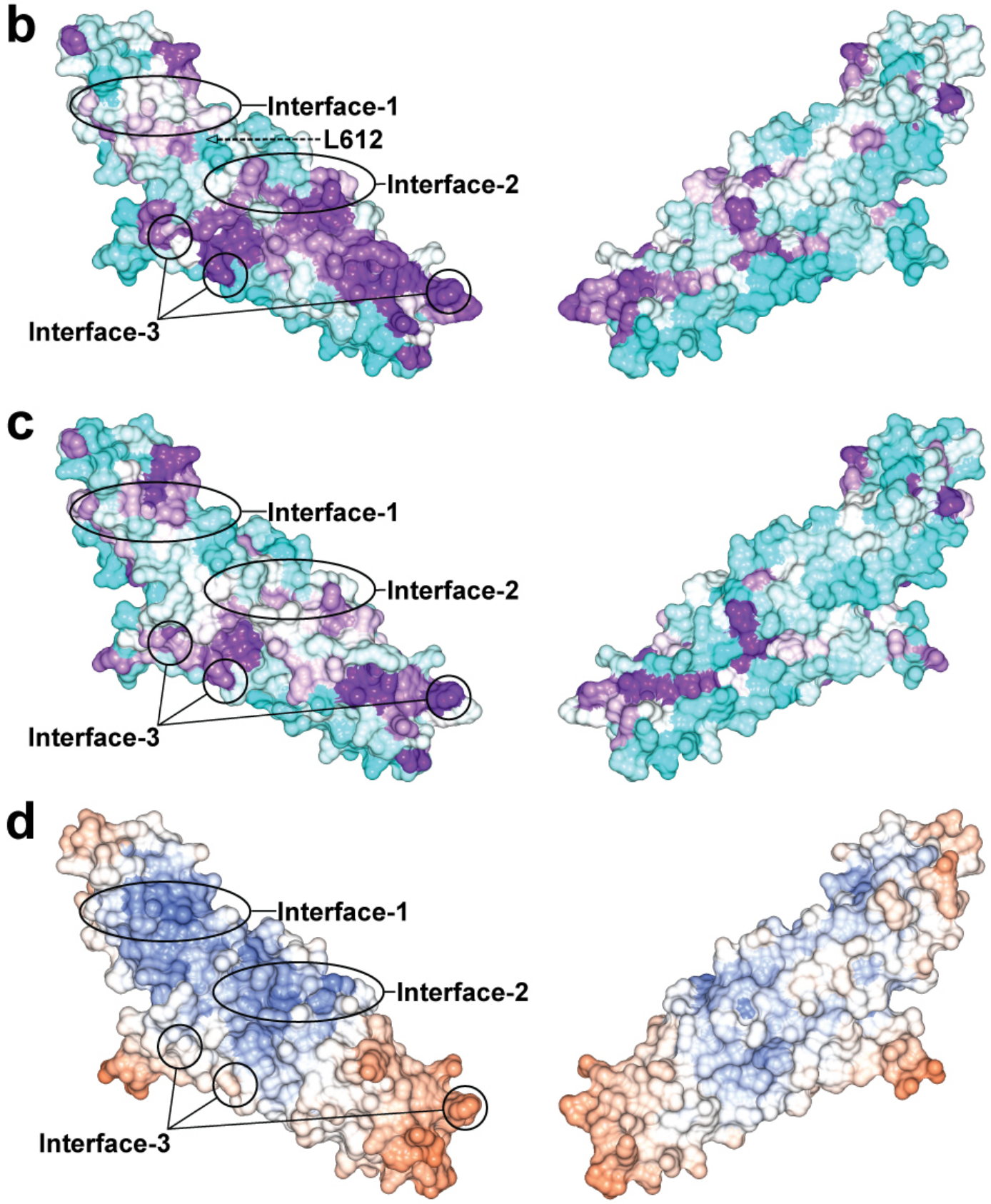

Supplementary Figure 5 


\section{Supplementary Figure 5: Sequence conservation within the stalk region}

a) Ribbon-type representation of the $\mathrm{MxA}$ stalk in two orientations, with selected residues of the interfaces shown in ball-and-stick.

b) Sequence conservation within the Mx family. Sequence conservation surface plot of the stalk of MxA in the same orientations as in (a) where conserved residues are shown in purple and non-conserved residues in cyan. Sequences of $33 \mathrm{Mx}$ proteins of different species have been used to detect conservation. The approximate position of Leu612 which has previously been reported as critically involved in MxA oligomerization is indicated $^{58,59}$. Leu612 is completely buried in between interface- 1 and 2 and, according to our structure, does not directly participate in inter-molecular interactions but contributes to the hydrophobic core of the stalk. Consequently, its mutation to lysine might result in unspecific destabilization of the stalk architecture.

c) Sequence conservation between $\mathrm{Mx}$ and dynamins. Sequence conservation surface plot of the stalk of MxA in the same orientations as in (a) where conserved residues are shown in purple and non-conserved residues in cyan. The alignment in Supp. Fig. 3 with six Mx and six dynamin sequences was used to detect conserved surface patches in these two families.

d) Surface representation of the MxA stalk, in which low temperature (B-) factors are represented in light blue and high temperature factors in red. Interface-1 and 2 appear to be stabilised by oligomerization (indicated by low B-factors), whereas interface-3 shows higher temperature factors pointing to increased flexibility in this region. In a ring-like oligomer, interface-3 might be stabilised by additional contacts (see Fig. 4, Supp. Fig. 12). 

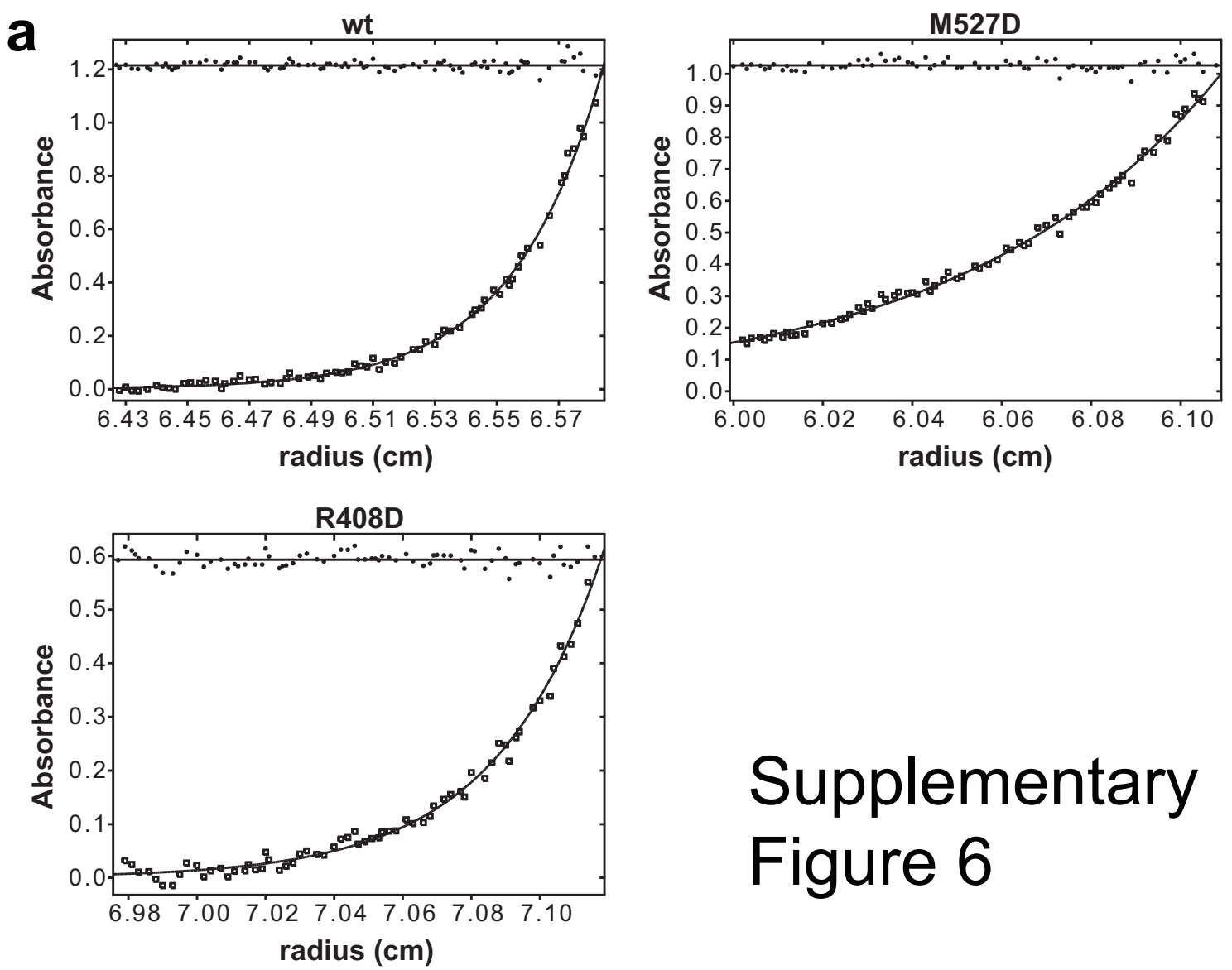

\section{Supplementary Figure 6}
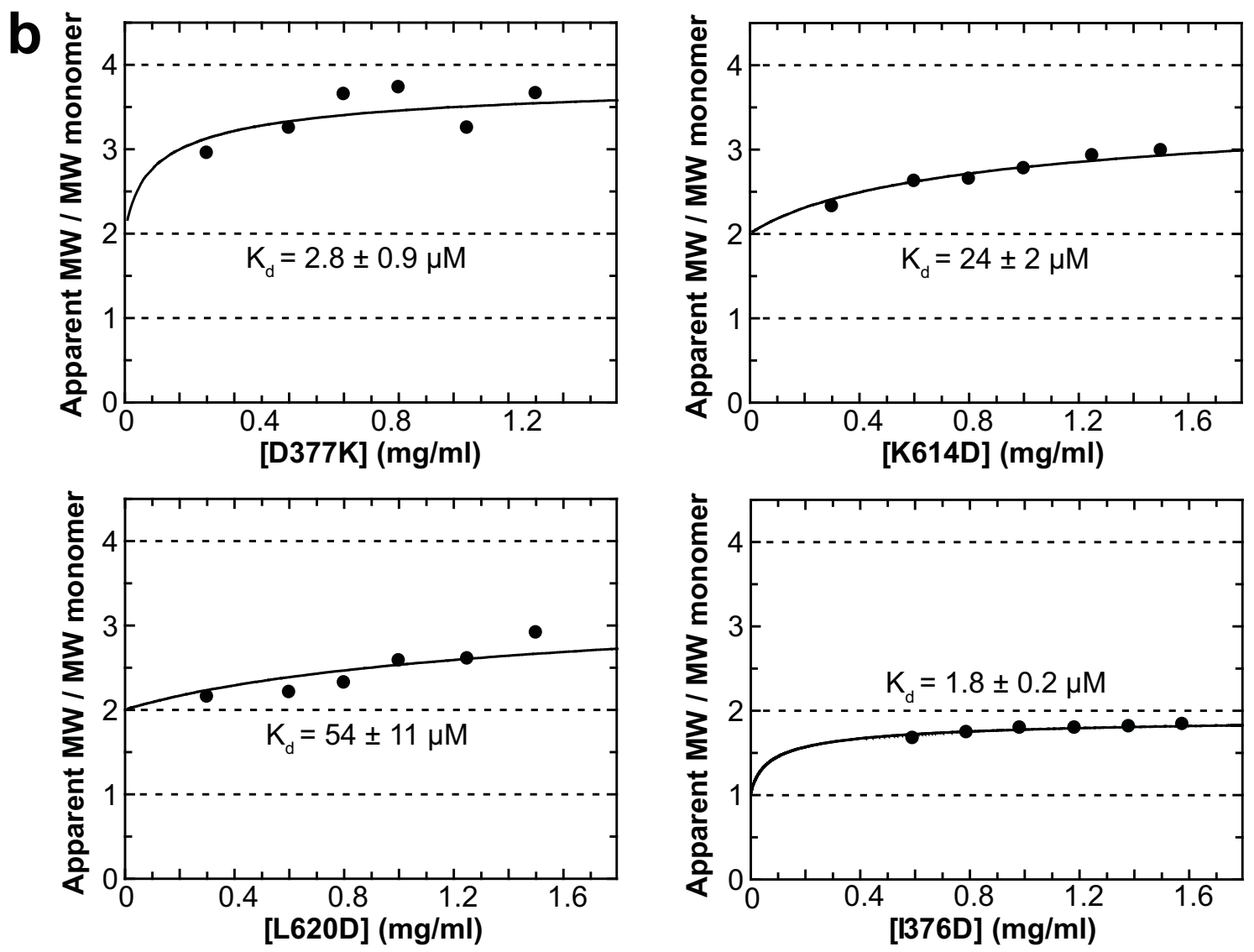

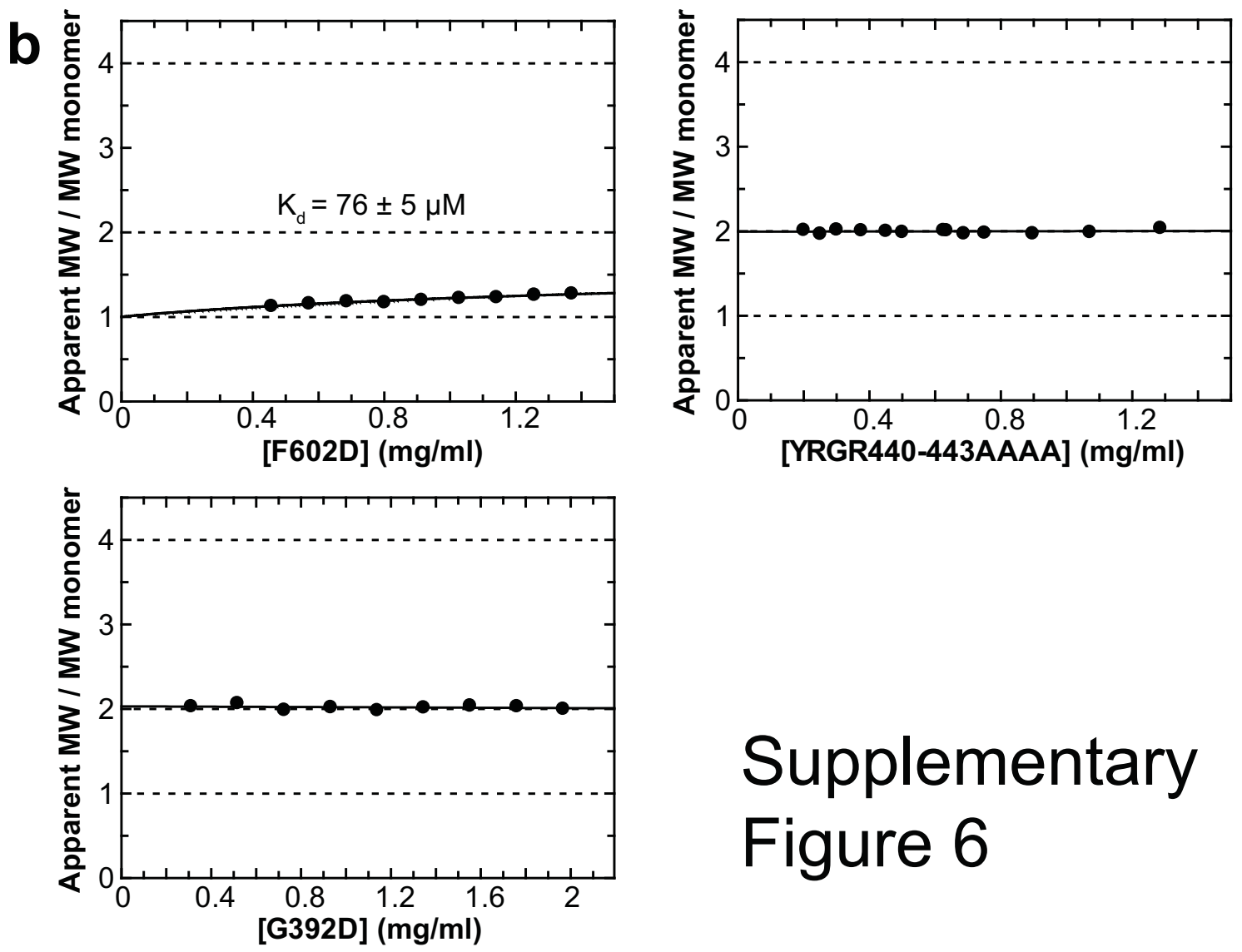

\section{Supplementary \\ Figure 6}
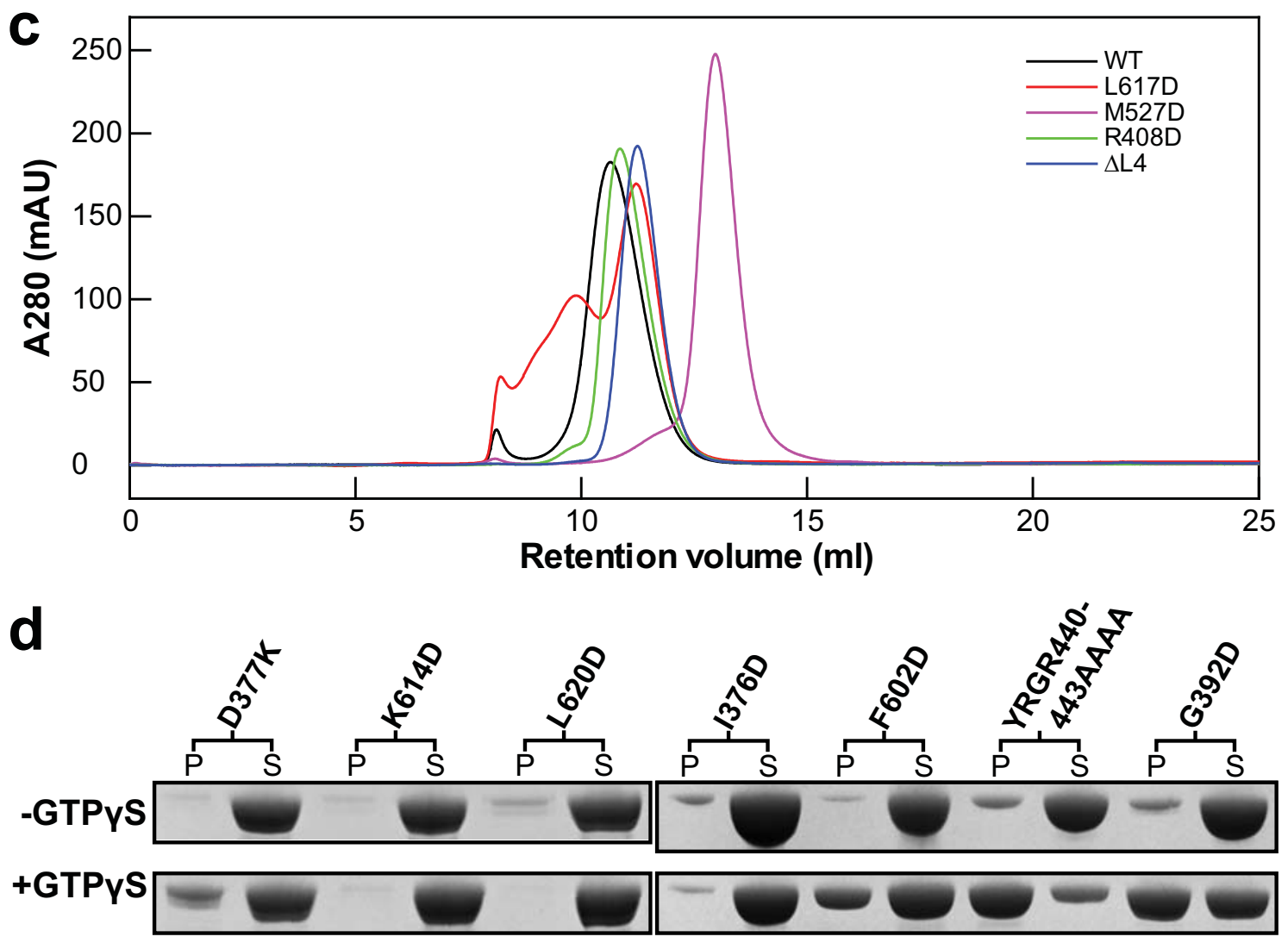


\section{Supplementary Figure 6: Assembly of MxA}

a) Representative data fittings of sedimentation equilibrium experiments for wt MxA, M527D and R408D (all at $1 \mathrm{mg} / \mathrm{ml}$ ), with residuals of the fit shown on top. The following apparent molecular weights were obtained from the data fittings: wt MxA: $320 \mathrm{kD} \pm 11 \mathrm{kD} . \mathrm{M} 527 \mathrm{D}: 94 \mathrm{kD} \pm 2 \mathrm{kD}$. R408D: $154 \mathrm{kD} \pm 4 \mathrm{kD}$.

b) Analytical ultracentrifugation runs in the absence of nucleotide at $400 \mathrm{mM} \mathrm{NaCl}$, as described in Methods. The following values were obtained from the data fitting: D377K: dimer-tetramer equilibrium, $\mathrm{K}_{\mathrm{d}}=2.8 \mu \mathrm{M} \pm 0.9 \mu \mathrm{M}$. K614D: dimer-tetramer equilibrium, $\mathrm{K}_{\mathrm{d}}=24 \mu \mathrm{M} \pm 2 \mu \mathrm{M}$. L620D: dimer-tetramer equilibrium, $\mathrm{K}_{\mathrm{d}}=54 \mu \mathrm{M} \pm$ $11 \mu \mathrm{M}$, I376D: monomer-dimer equilibrium, $\mathrm{K}_{\mathrm{d}}=1.8 \mu \mathrm{M} \pm 0.2 \mu \mathrm{M}$. F602D: monomerdimer equilibrium, $\mathrm{K}_{\mathrm{d}}=76 \mu \mathrm{M} \pm 5 \mu \mathrm{M}$. YRGR440-443AAAA: stable dimer. G392D: stable dimer. Mutations in the centre of interface-1 (I376D, L617D) have a more severe impact on the native assembly of MxA than mutations in the periphery of interface-1 (D377K, K614D, L620D).

c) $1 \mathrm{mg}$ of wt MxA or the indicated mutants was applied to a Superdex200 10/300 analytical gel filtration column using a buffer containing 20 mM HEPES pH 7.5, 400 $\mathrm{mM} \mathrm{NaCl}, 2 \mathrm{mM} \mathrm{MgCl} 2,2.5 \mathrm{mM}$ DTT. Wt MxA (black) eluted as a tetramer from gel filtration whereas mutants L617D (red) in interface-1, R408D (green) in interface-3 and $\Delta$ L4 (blue) eluted slightly later. Mutant M527D (magenta) eluted as a monomer. Note that mutants in interface-1 (also I376D) eluted in several peaks in these experiments, possibly indicating that these mutations partially destabilise the protein.

d) Oligomerization assays for mutants D377K, K614D, L620D, I376D, F602D, YRGR440-443AAAA and G392D were carried out under the same conditions as in Fig. 2b. I376D, K614D and L620D in interface-1 did not form oligomers, both in the presence and absence of GTP- $\gamma-\mathrm{S}$. D377K in interface-1, F602D in interface-2 and YRGR440-443AAAA, G392D in interface-3 could partly form oligomers only in the presence of GTP- $\gamma-\mathrm{S}$. 


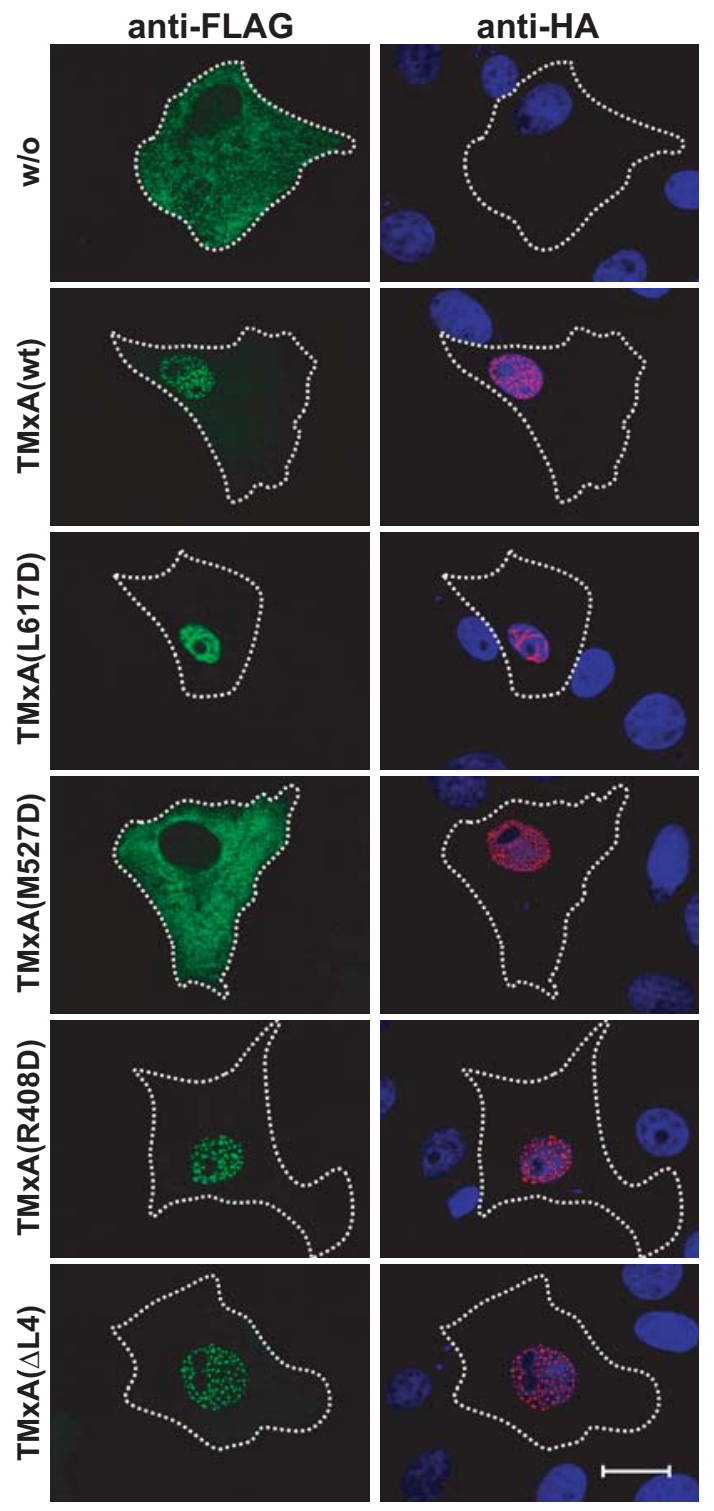

\section{Supplementary Figure 7: Nuclear translocation assay}

TMxA, an artificial nuclear form of MxA carrying the SV40 large T nuclear localization signal and an HA-tag, and the indicated TMxA mutants were co-expressed with FLAGtagged wt MxA in Vero E6 cells. $20 \mathrm{~h}$ after transfection, cells were fixed and stained with monoclonal antibodies directed against the HA-tag (red) and the FLAG-tag (green). A DAPI nuclear staining (blue) is overlayed with the HA staining. In cotransfected cells, the fluorescence intensity of FLAG-tagged wt MxA in cytoplasm and nucleus was quantified using the Axiovision software (Zeiss) (Fig. 2c, $n=20$ for each experiment). 

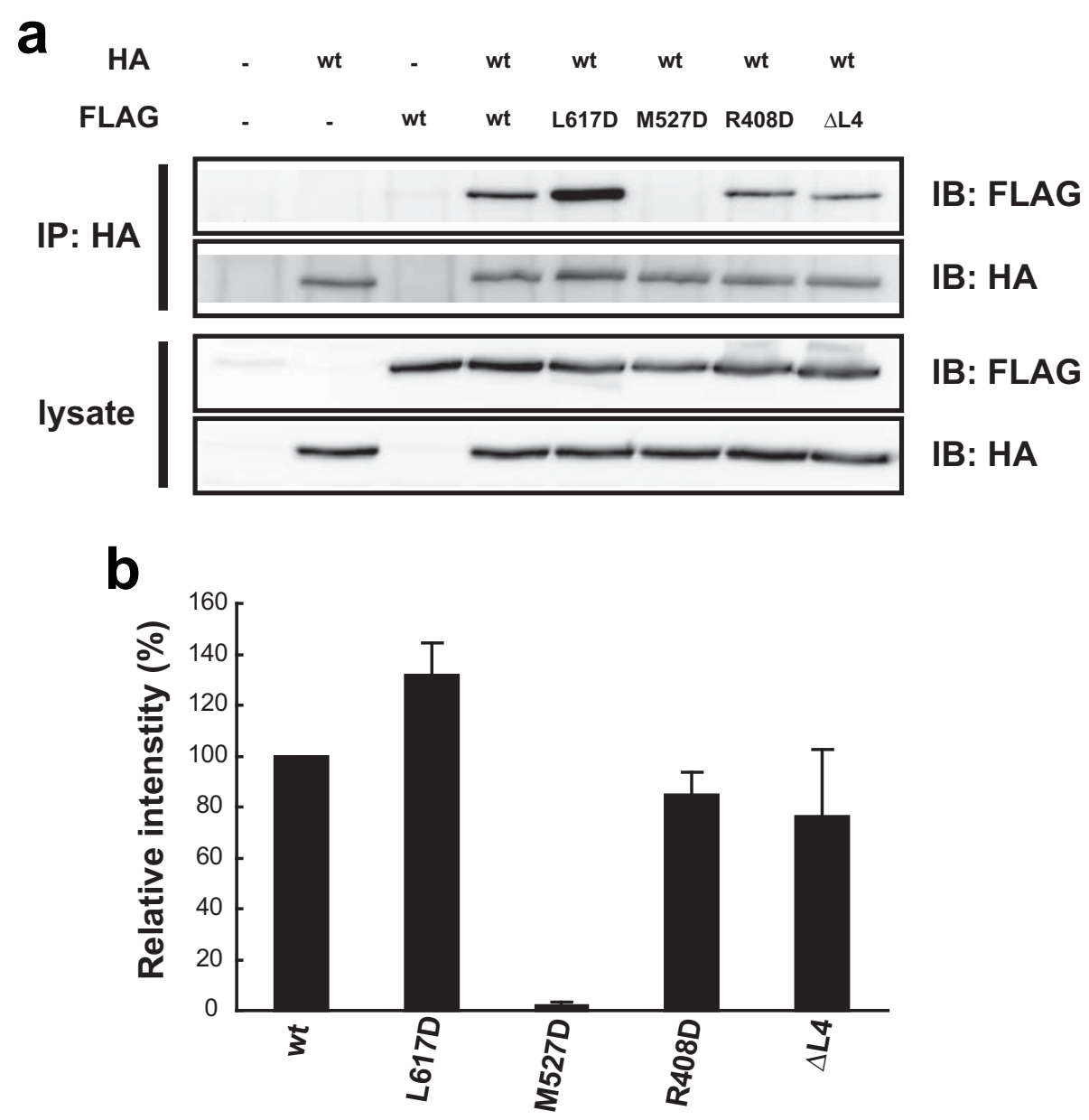

Supplementary Figure 8: Co-immunoprecipitation studies of wt MxA with the indicated MxA mutants

a) $293 \mathrm{~T}$ cells were co-transfected with $1.5 \mu \mathrm{g}$ expression plasmids encoding the indicated HA-tagged wt MxA and FLAG-tagged wt or mutant MxA constructs. After $24 \mathrm{~h}$, cells were lysed and HA-tagged wt MxA was immunoprecipitated. After extensive washing, samples were subjected to SDS-PAGE and western blotting with antibodies directed against the HA- and FLAG-tag. Lysates represent $5 \%$ of the total input.

b) Western blot quantification of co-immunoprecipated FLAG-tagged wt MxA and MxA mutants was done with the Quantity One software (Bio-Rad). Shown are the mean and the standard deviation of three independent experiments. 


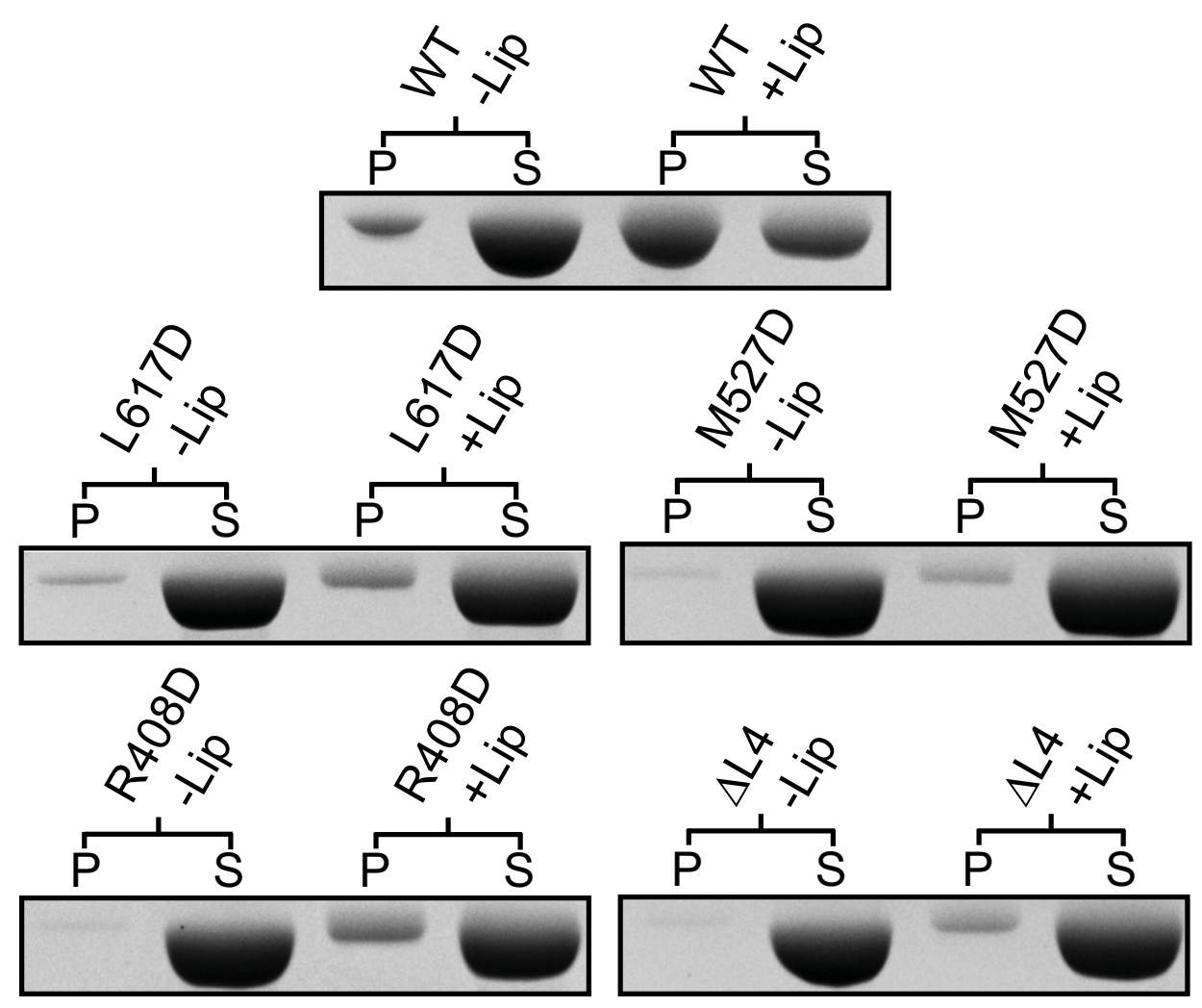

\section{Supplementary Figure 9: Liposome binding of MxA and mutants}

Folch liposome (lipids derived from bovine brain) co-sedimentation assays for wt MxA and the indicated mutants at a protein concentration of $0.75 \mathrm{mg} / \mathrm{ml}$. To measure exclusively binding/oligomerization of $\mathrm{MxA}$ at the liposome surface and to avoid oligomerization in the absence of liposomes, the salt concentration was optimised to $300 \mathrm{mM} \mathrm{NaCl}$. Under these conditions, none of the mutants bound to liposomes. $\mathrm{P}$ pellet fraction. S - supernatant. 
a
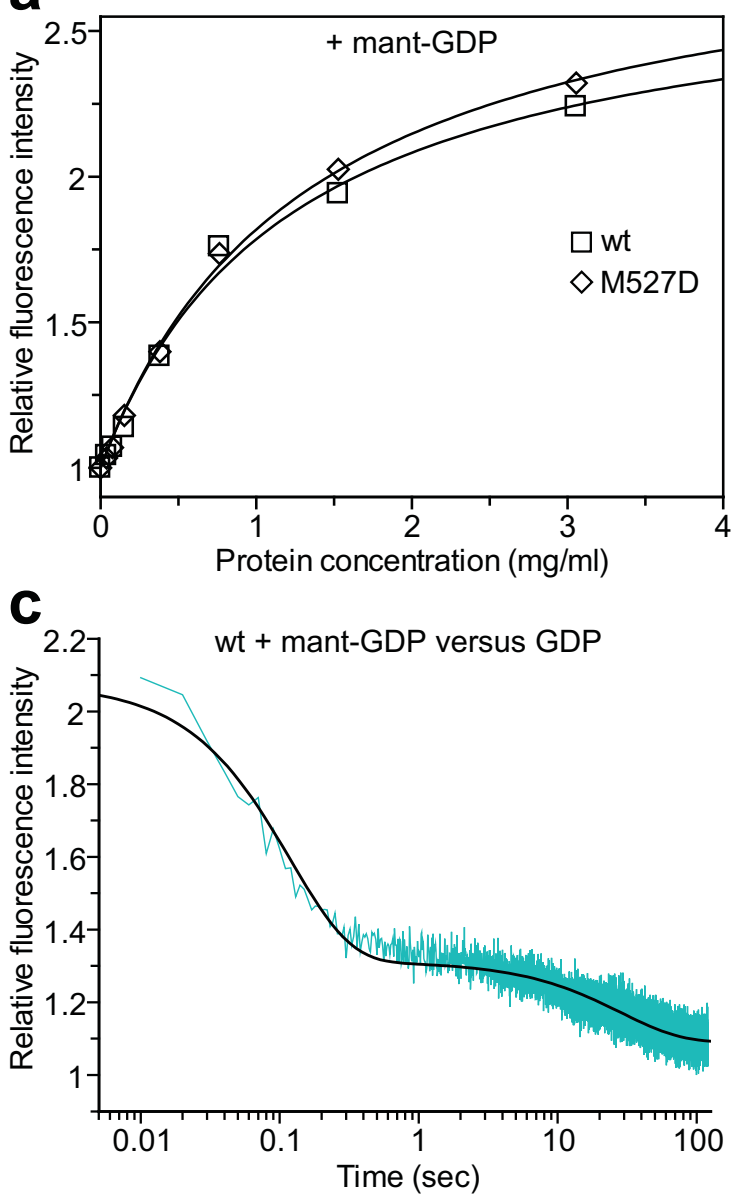

d

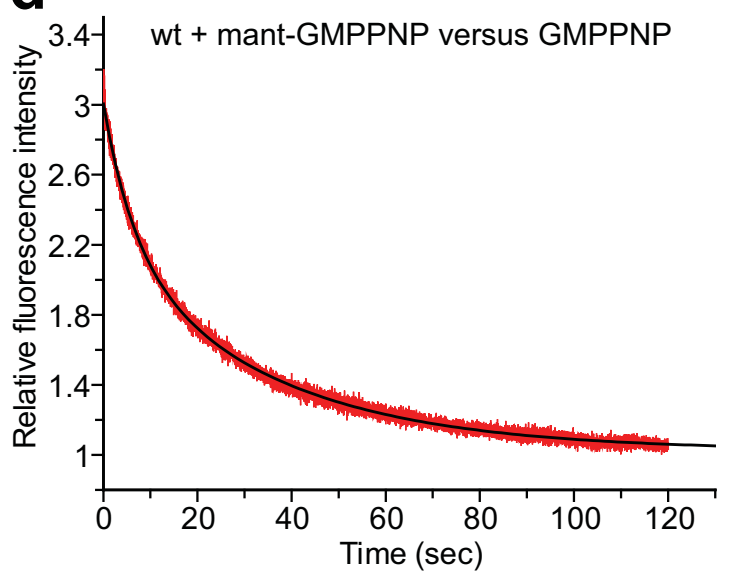

b
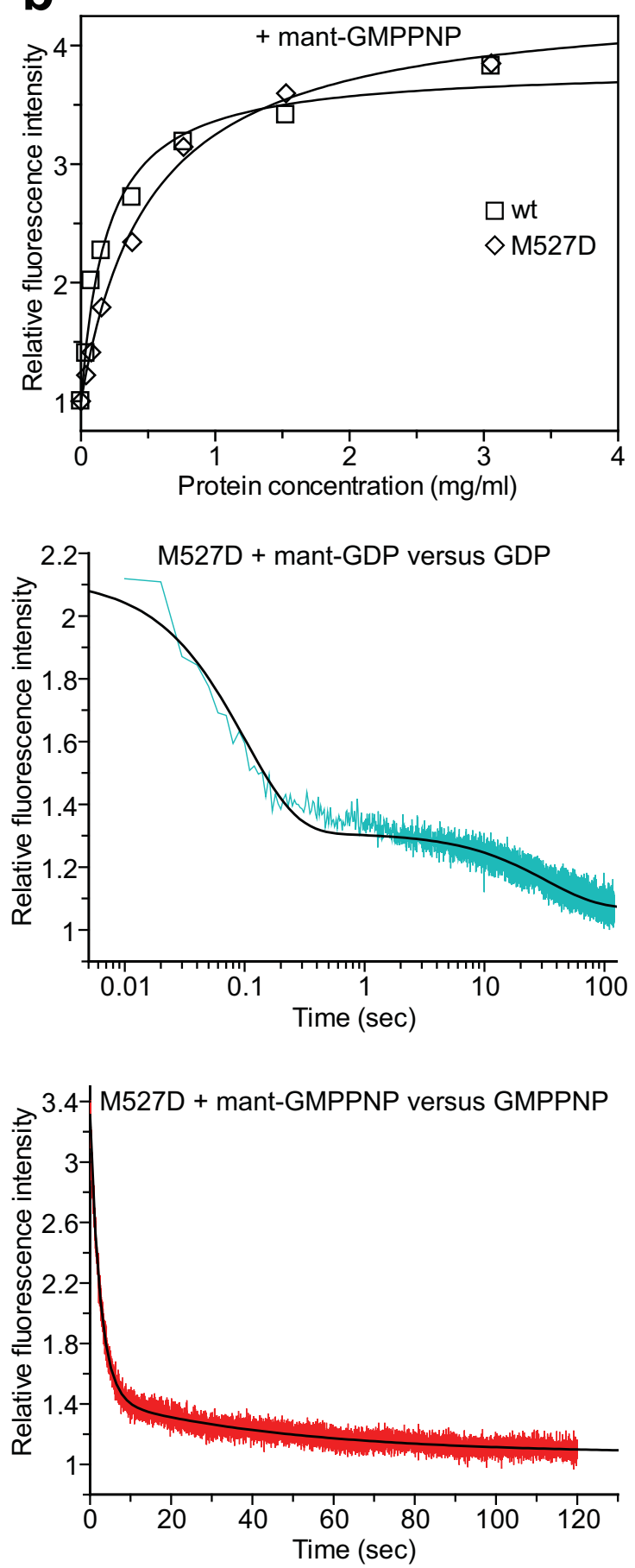

Supplementary Figure 10 


\section{Supplementary Figure 10: Nucleotide binding characteristics of MxA}

a) $1 \mu \mathrm{M}$ 2'/3'-O-(N-Methyl-anthraniloyl)(mant-)GDP, a fluorophore-coupled GDP analogue (Jena Bioscience), or (b) $1 \mu \mathrm{M}$ mant-GMPPNP, a non-hydrolysable flourophore-coupled GTP analogue, were incubated in GTPase reaction buffer at $37^{\circ} \mathrm{C}$ with increasing concentrations of wt MxA and the M527D mutant. The peak fluorescence of an emission spectrum, recorded at an FP-6500 fluorescence spectrometer (Jasco) for each protein concentration, was plotted against the protein concentration (excitation wavelength $366 \pm 4 \mathrm{~nm}$, according to ref. 24). Protein binding resulted in de-quenching of mant-fluorescence, concomitant with a fluorescence increase. Whereas wt MxA and the M527D mutant bound with similar affinities to mant-GDP $\left(\mathrm{K}_{\mathrm{d}}=16 \mu \mathrm{M}\right.$ for wt and $\mathrm{K}_{\mathrm{d}}=18 \mu \mathrm{M}$ for M527D), wt MxA showed a 3-fold higher apparent affinity for mant-GMPPNP $\left(\mathrm{K}_{\mathrm{d}}=2.3 \mu \mathrm{M}\right)$ compared to the M527D mutant $\left(\mathrm{K}_{\mathrm{d}}=6.8 \mu \mathrm{M}\right)$.

c,d) In stopped-flow experiments, using an RX2000 Rapid Kinetics Spectrometer Accessory (Applied Photophysics) coupled to an Aminco Bowman Series 2 spectrofluorometer, the off-rates for mant-GDP (c) and mant-GMPPNP (d) were determined at $37^{\circ} \mathrm{C}$ by following the fluorescence after rapid mixing of $4 \mu \mathrm{M}$ mantnucleotide, $40 \mu \mathrm{M}$ wt MxA or the M527D mutant in one syringe and a 1000-fold excess of unlabelled nucleotide (GDP or GMPPNP, respectively) in the second syringe (excitation wavelength $366 \pm 4 \mathrm{~nm}$, measured emission at $435 \pm 16 \mathrm{~nm}$ ). A double exponential decay of fluorescence was observed for both reactions. The slower rate was not caused by bleaching of the mant-fluorophore. Wt MxA and M527D showed similar off-rates for mant-GDP $\left(\mathrm{k}_{\mathrm{off1}}=503 \mathrm{~min}^{-1}, \mathrm{k}_{\mathrm{off} 2}=2.1 \mathrm{~min}^{-1}\right.$ for $\mathrm{wt} \mathrm{MxA}$ and $\mathrm{k}_{\text {off } 1}=595 \mathrm{~min}^{-1}, \mathrm{k}_{\text {off2 }}=1.8 \mathrm{~min}^{-1}$ for M527D). The first observed off-rate, $\mathrm{k}_{\text {off } 1}$, is fast and accounts for $75 \%$ of the fluorescent decay, whereas the second observed off-rate, $\mathrm{k}_{\mathrm{off}}$, is 250 -fold slower. $\mathrm{k}_{\text {off } 1}$ for both wt MxA and M527D is much faster than the GTP 
turnover suggesting that GDP release is not the rate-limiting step in the GTPase reaction. Due to the fast fluorescence decrease, the $\mathrm{x}$-axis is shown in logarithmic scale.

For mant-GMPPNP, $\mathrm{k}_{\text {off1 }}$ was slower compared to mant-GDP. Furthermore, $\mathrm{k}_{\text {off1 }}$ for mant-GMPPNP was 2.6-fold slower for wt MxA $\left(\mathrm{k}_{\text {off } 1}=9.2 \mathrm{~min}^{-1}, \mathrm{k}_{\text {off } 2}=1.8 \mathrm{~min}^{-1}\right)$ than for the M527D mutant $\left(\mathrm{k}_{\text {off1 }}=24 \mathrm{~min}^{-1}, \mathrm{k}_{\mathrm{off} 2}=1.3 \mathrm{~min}^{-1}\right)$ which would account for the observed affinity difference for GMPPNP. The deduced on-rates for GMPPNP are approximately $4 \min ^{-1} \mu \mathrm{M}^{-1}$ and are in a similar range as previously described ${ }^{24}$. In the GTPase assays in the presence of $1 \mathrm{mM}$ GTP, the GTP binding rates are therefore fast (around $4000 \mathrm{~min}^{-1}$ ) and not rate-limiting.

$\mathrm{k}_{\text {off } 1}$ for mant-GMPPNP closely approaches the maximal GTPase turnover number at high protein concentrations for both wt MxA and M527D. Based on these results, we suggest a model for the GTPase reaction, where at low protein concentrations, selfassembly of MxA via the G-domains is limiting for the GTPase reaction in solution, resulting in a similar increase in $\mathrm{k}_{\mathrm{obs}}$ with increasing protein concentrations for each mutant. At higher protein concentrations/higher GTPase turnover, the off-rates for GDP-inorganic phosphate $\left(\mathrm{P}_{\mathrm{i}}\right)$ after GTP hydrolysis or conformational changes associated with this step might be rate-limiting, as for example in the myosin system, where also the release of inorganic $\mathrm{P}_{\mathrm{i}}$ is rate-limiting ${ }^{60}$. Similarly as for mant-GMPPNP, these off rates might be slower for wt MxA than for the monomeric/dimeric mutants resulting in the observed differences in GTPase rates at higher protein concentrations. 

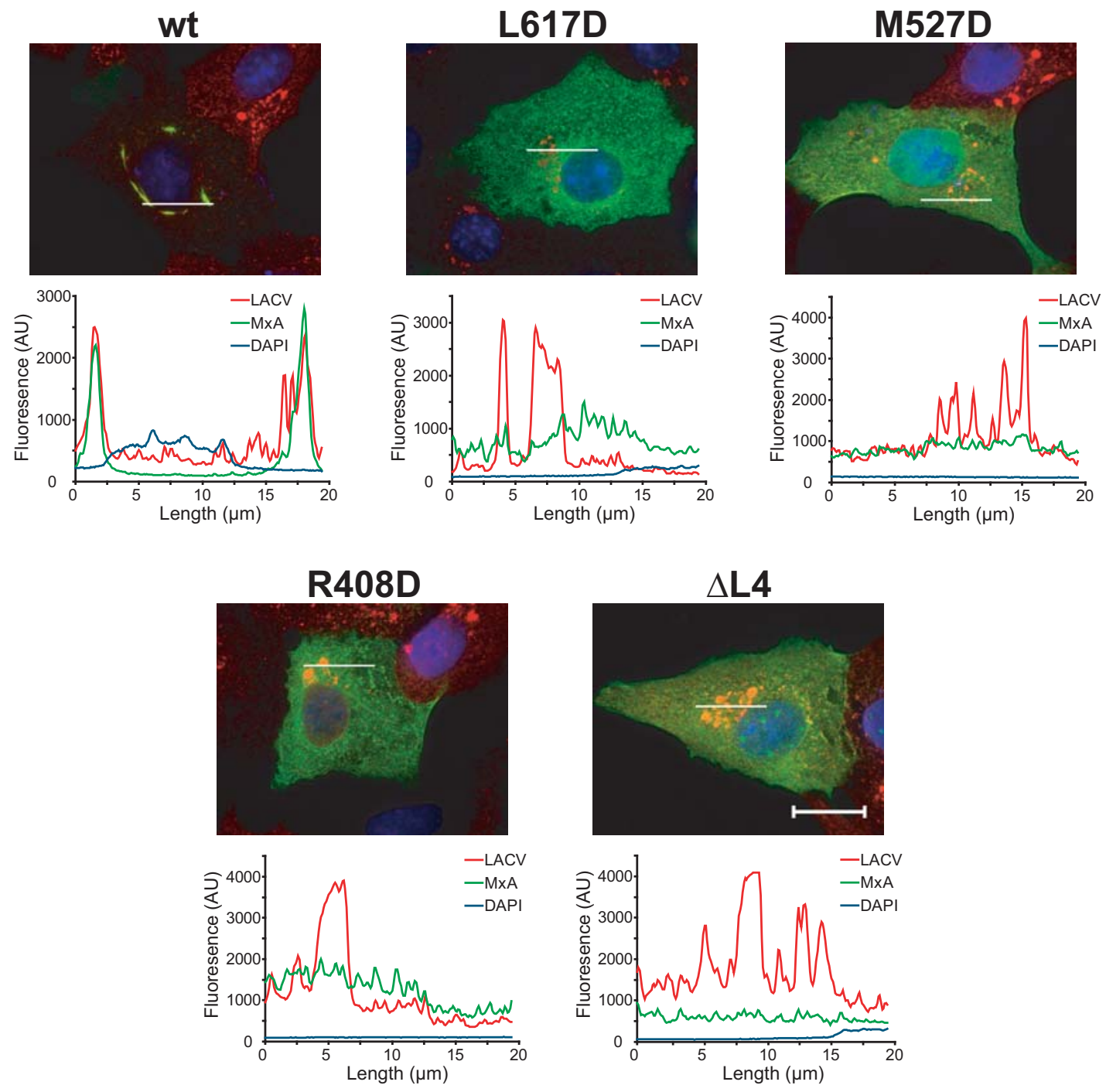

\section{Supplementary Figure 11: Quantification of the $\mathbf{M x A} / \mathbf{N}$ positive complexes}

Formation of $\mathrm{MxA} / \mathrm{N}$ protein complexes was quantified by generating fluorescence intensity profiles across nucleocapsid protein positive complexes as indicated in the figure. The intensities of the green and red signals for MxA and N, respectively, were determined using the Axiovision software (Zeiss, Jena). Co-localization of MxA and $\mathrm{N}$ was considered when overlapping peaks were observed, as exemplarily shown for wt MxA. 97\% of infected cells expressing wt MxA but none of the cells expressing mutant MxA constructs $(n=100)$ showed MxA/N complex formation. Intensity is given in arbitrary units (AU) (bar=20 $\mu \mathrm{m})$. 


\section{a}

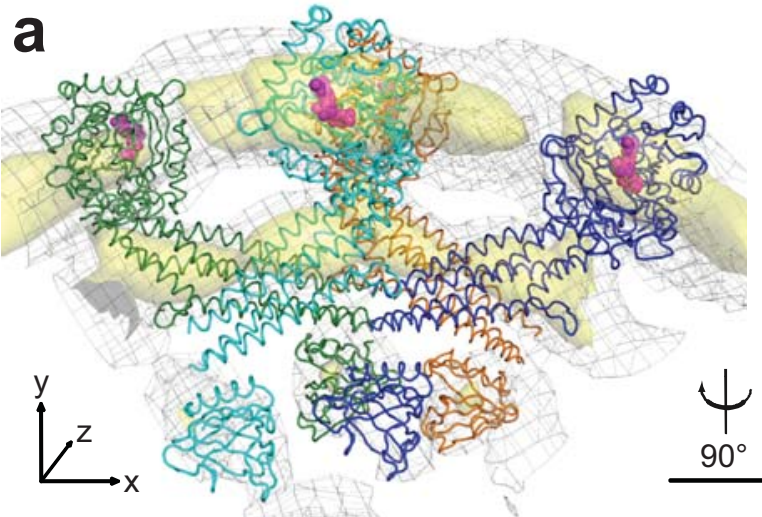

C

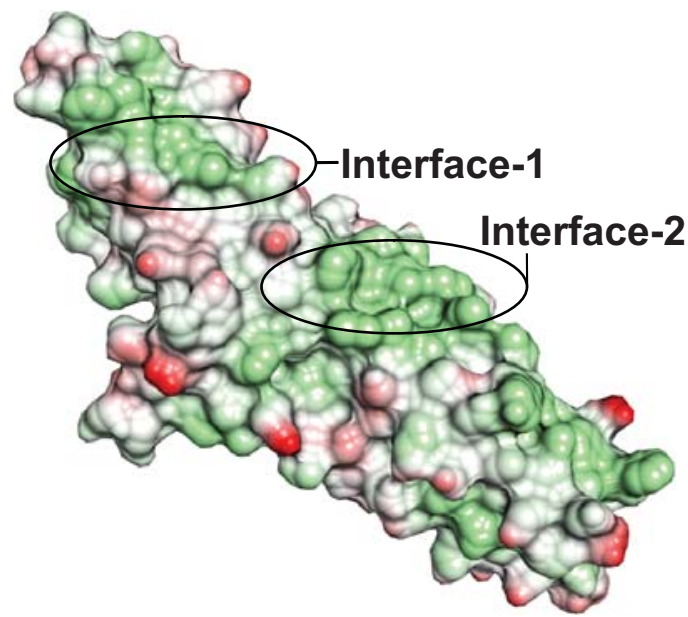

e

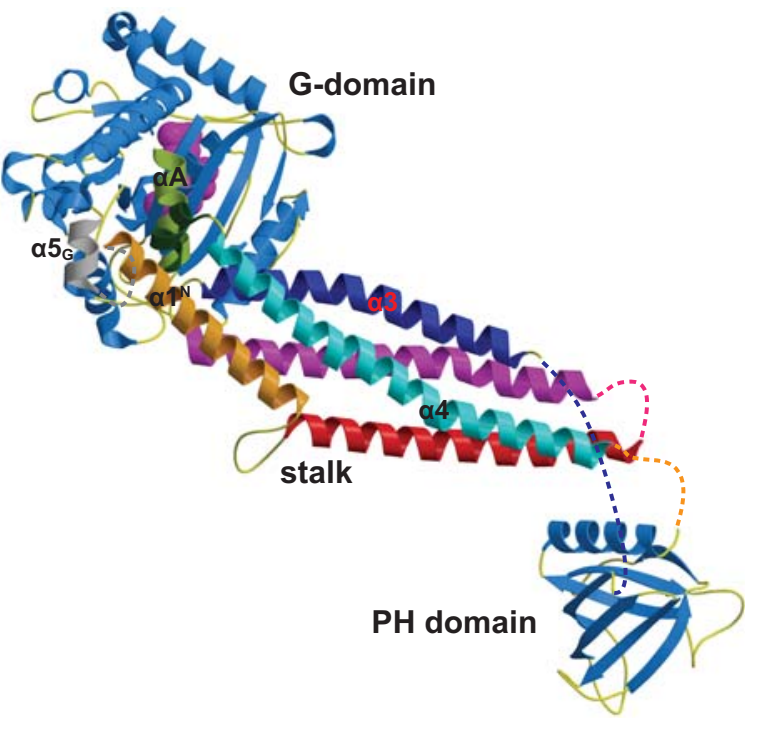

b

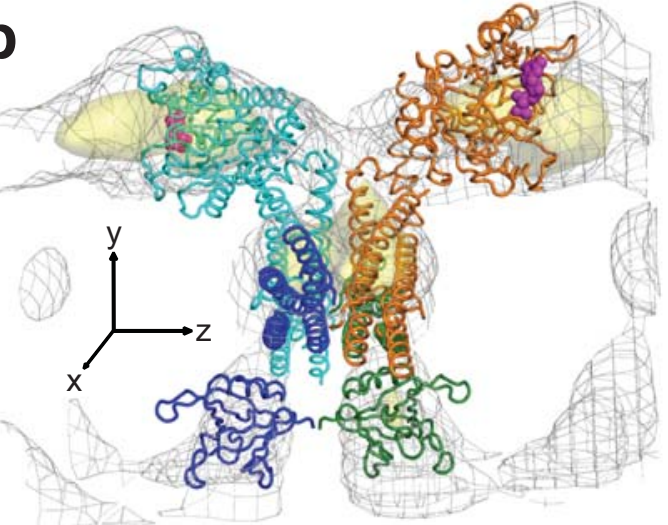

d
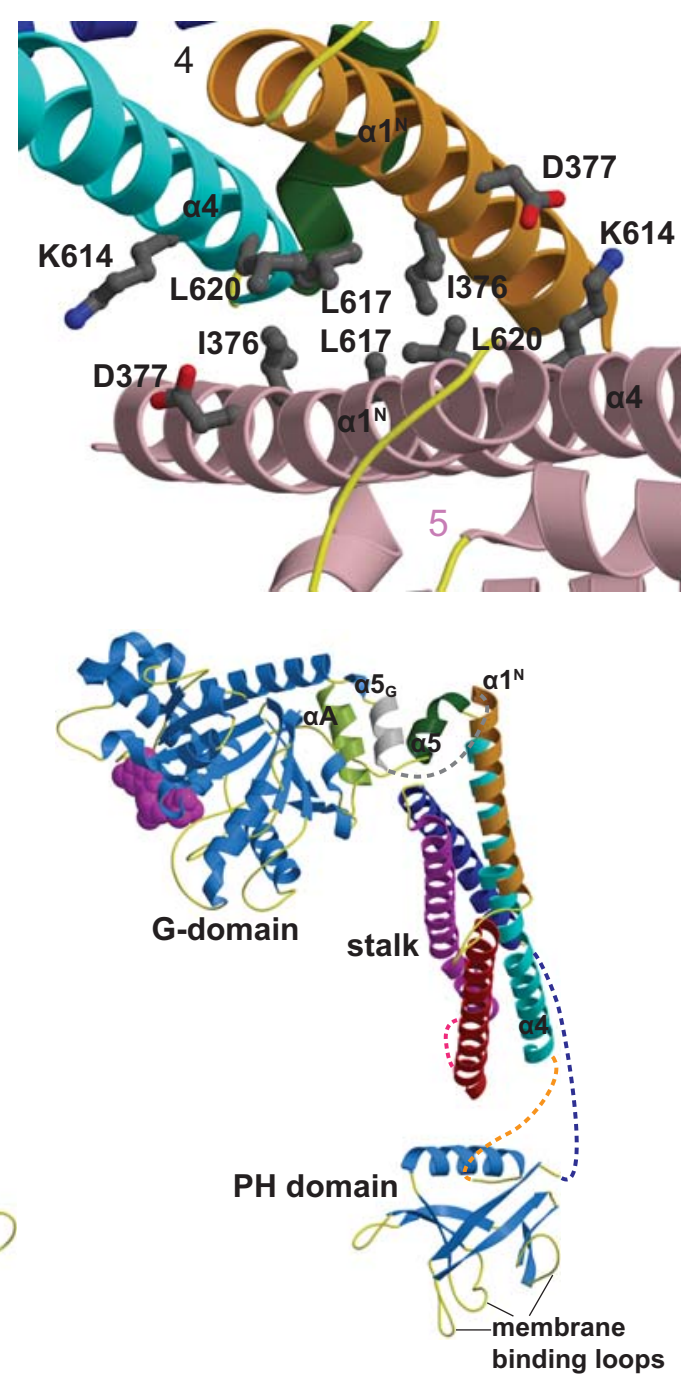

Supplementary Figure 12 


\section{Supplementary Figure 12: Construction of the dynamin oligomer}

a) Two stalk dimers, each associated via interface-2, were rotated against each other around interface-1 and fitted into the cryo-EM map of oligomerised, nucleotide-bound $\triangle \mathrm{PRD}$ dynamin in the constricted state ${ }^{5}$. The orientations of the nucleotide-free Gdomains of rat dynamin were adapted from earlier fittings ${ }^{5}$. The position of the nucleotide (in magenta) within the G-domain is derived from the crystal structure of the GDP-bound Dictyostelium discoideum dynamin G-domain ${ }^{61}$. The PH domains of dynamin $^{52,62}$ were fitted into the density, with the membrane binding loops ${ }^{55,56}$ directed towards the tubulated liposome and the $\mathrm{N}$ - and $\mathrm{C}$-termini directed towards the stalk region. Electron density is represented by a gray mesh at contour level $1.5 \sigma$ and by a yellow surface at contour level 3.5 $\sigma$. In a front view (b), the T-bar shape of our model becomes apparent which is in good agreement with the electron density. Only two Gand PH domains are shown for better clarity.

c) Surface representation of interface-1 with hydrophobic atoms in green and charged residues in red. The main driving force for assembly of interface-1 is likely the coverage of hydrophobic residues which is fully maintained by the proposed rotation.

d) Interface-1 in the "rotated" dynamin oligomer, with key residues as in Fig. 1d. All residues shown to be important for assembly (Fig. 2a, Supp. Fig. 6) are still involved in this interface after the rotation. Also the salt bridge between Asp377 and Lys614 could be preserved, due to the long flexible side chain of Lys614.

e) Two views on our monomeric dynamin model showing the proposed connection between G-domain and stalk (grey loop) and between stalk and the PH domain (magenta and orange loops). The C-terminal helix $\alpha 5_{\mathrm{G}}$ of the G-domain is coloured in grey whereas helix $\alpha \mathrm{A}$ at the $\mathrm{N}$-terminus of the G-domain is coloured in light green. For dynamin, it has been proposed that these two helices together with the C-terminal region 
of the GED not resolved in our structure form a helical bundle, the "bundle signalling element"13 ${ }^{13}$ and our model would be consistent with this hypothesis. The predicted loops connecting stalk and $\mathrm{PH}$ domain are both approximately 30 residues long which would allow the suggested connection. 
Supplementary Table 1: Data collection statistics

\begin{tabular}{|c|c|c|}
\hline & native & selenomethionine-derivative \\
\hline \multicolumn{3}{|l|}{ Data collection } \\
\hline Space group & $\mathrm{P} 1$ & $\mathrm{P} 1$ \\
\hline \multicolumn{3}{|l|}{ Cell dimensions } \\
\hline$a, b, c(\AA)$ & $50.1,57.4,57.9$ & $51.0,57.7,57.5$ \\
\hline$\alpha, \beta, \gamma\left({ }^{\circ}\right)$ & $65.8,88.4,74.8$ & $65.6,88.9,75.2$ \\
\hline Wavelength $(\AA)$ & 0.91841 & 0.97968 \\
\hline Resolution $(\AA)$ * & $19.95-2.40(2.46-2.40)$ & $19.74-2.76(2.83-2.76)$ \\
\hline$R_{\text {sym }} *$ & $0.062(0.387)$ & $0.067(0.395)$ \\
\hline$|/ \sigma|^{*}$ & $14.91(3.76)$ & $7.92(2.08)$ \\
\hline Completeness $(\%)^{*}$ & $97.1(96.7)$ & $93.2(86.6)$ \\
\hline Redundancy & $3.52(3.54)$ & $1.99(1.95)$ \\
\hline
\end{tabular}

*Numbers in brackets represent values from the highest resolution shell. 
Supplementary Table 2: Refinement statistics

\begin{tabular}{|c|c|}
\hline & native \\
\hline Resolution $(\AA)$ & $19.95-2.40$ \\
\hline No. reflections & 20,309 \\
\hline$R_{\text {work }} / R_{\text {free }}$ & $0.193 / 0.257$ \\
\hline Molecules / asymmetric unit & 2 \\
\hline \multicolumn{2}{|l|}{ Number of atoms } \\
\hline Protein & 3,677 \\
\hline Ligand/ion & 0 \\
\hline Water & 102 \\
\hline \multicolumn{2}{|l|}{ B-factors $\left(\AA^{2}\right)$} \\
\hline Protein & 45 \\
\hline Water & 24 \\
\hline \multicolumn{2}{|l|}{ R.m.s. deviations } \\
\hline Bond lengths $(\AA)$ & 0.011 \\
\hline Bond lengths $(\AA)$ & 1.152 \\
\hline
\end{tabular}




\section{Supplementary Notes: References of the Supplementary Material}

50 Thompson, J. D., Higgins, D. G., and Gibson, T. J. CLUSTAL W: improving the sensitivity of progressive multiple sequence alignment through sequence weighting, position-specific gap penalties and weight matrix choice. Nucleic Acids Res. 22, 4673-4680 (1994)

51 Cole, C., Barber, J. D., and Barton, G. J. The Jpred 3 secondary structure prediction server. Nucleic Acids Res. 36, W197-W201 (2008)

52 Ferguson, K. M., et al. Crystal structure at 2.2 A resolution of the pleckstrin homology domain from human dynamin. Cell 79, 199-209 (1994)

53 Wallace, A. C., Laskowski, R. A., and Thornton, J. M. LIGPLOT: a program to generate schematic diagrams of protein-ligand interactions. Protein Eng 8, 127134 (1995)

54 Jensen, R. E., et al. Yeast mitochondrial dynamics: fusion, division, segregation, and shape. Microsc. Res. Tech. 51, 573-583 (2000)

Vallis, Y., et al. Importance of the pleckstrin homology domain of dynamin in clathrinmediated endocytosis. Curr. Biol. 9, 257-260 (1999)

56 Zheng, J., et al. Identification of the binding site for acidic phospholipids on the $\mathrm{pH}$ domain of dynamin: implications for stimulation of GTPase activity. J Mol Biol. 255, 14-21 (1996)

57 Prakash, B., et al. Triphosphate structure of guanylate-binding protein 1 and implications for nucleotide binding and GTPase mechanism. EMBO J 19, 45554564 (2000)

Schumacher, B. and Staeheli, P. Domains mediating intramolecular folding and oligomerization of MxA GTPase. J Biol. Chem. 273, 28365-28370 (1998)

Janzen, C., Kochs, G., and Haller, O. A monomeric GTPase-negative MxA mutant with antiviral activity. $J$ Virol. 74, 8202-8206 (2000)

Pollard, T. D. and Ostap, E. M. The chemical mechanism of myosin-I: implications for actin-based motility and the evolution of the myosin family of motor proteins. Cell Struct. Funct. 21, 351-356 (1996)

61 Niemann, H. H., et al. Crystal structure of a dynamin GTPase domain in both nucleotide-free and GDP-bound forms. EMBO J 20, 5813-5821 (2001)

62 Timm, D., et al. Crystal structure of the pleckstrin homology domain from dynamin. Nat Struct. Biol. 1, 782-788 (1994) 